\title{
Scaling Invariants and Symmetry Reduction of Dynamical Systems
}

\author{
Evelyne Hubert * $\quad$ George Labahn ${ }^{\dagger}$
}

February 10, 2012

\begin{abstract}
Scalings form a class of group actions that have theoretical and practical importance. A scaling is accurately described by a matrix of integers. Tools from linear algebra over the integers are exploited to compute their invariants and offer a scheme for the symmetry reduction of dynamical systems. A special case of the symmetry reduction algorithm applies to reduce the number of parameters in physical, chemical or biological models.
\end{abstract}

Keywords: Group actions, Rational invariants, Matrix normal form, Model reduction, Dimensional analysis.

AMS Classification: 08-04 12-04 14L30 15-04

\section{Introduction}

Consider the following predator-prey model

$$
\begin{aligned}
\frac{d n}{d t} & =n\left(r\left(1-\frac{n}{K}\right)-k \frac{p}{n+d}\right), \\
\frac{d p}{d t} & =s p\left(1-h \frac{p}{n}\right)
\end{aligned}
$$

which has six parameters, $r, K, k, s, h, d$, and $s$. Following [20, Section 3.4] one introduces nondimensional variables

$$
\mathfrak{s}=\frac{s}{r}, \mathfrak{k}=\frac{k}{r h}, \mathfrak{d}=\frac{d}{K}, \quad \mathfrak{t}=r t, \quad \text { and } \mathfrak{n}=\frac{n}{K}, \mathfrak{p}=\frac{h}{K} p
$$

so as to simplify the system into

$$
\begin{aligned}
& \frac{d \mathfrak{n}}{d \mathfrak{t}}=\mathfrak{n}(1-\mathfrak{n})-\mathfrak{k} \frac{\mathfrak{n} \mathfrak{p}}{\mathfrak{n}+\mathfrak{d}}, \\
& \frac{d \mathfrak{p}}{d \mathfrak{t}}=\mathfrak{s} \mathfrak{p}\left(1-\frac{\mathfrak{p}}{\mathfrak{n}}\right)
\end{aligned}
$$

where there are only three parameters left, $\mathfrak{s}, \mathfrak{h}, \mathfrak{k}$.

In order to obtain this reduced system algorithmically, which is one of the main points of this paper, one has to notice that the original dynamical system admits a scaling symmetry: it is invariant under any of the the following change of variables parameterized $(\eta, \mu, \nu)$ :

$$
\begin{aligned}
& t \rightarrow \eta t, \quad r \rightarrow \eta^{-1} r, \quad s \rightarrow \eta^{-1} s, \\
& n \rightarrow \mu n, \quad h \rightarrow \mu \nu^{-1} h \quad k \rightarrow \eta^{-1} \mu \nu^{-1} k, \\
& p \rightarrow \nu p, \quad K \rightarrow \mu K, \quad d \rightarrow \quad \mu d .
\end{aligned}
$$

*INRIA Méditerranée, 06902 Sophia Antipolis, France evelyne.hubert@inria.fr

${ }^{\dagger}$ Cheriton School of Computer Science, University of Waterloo, Waterloo ON, Canada N2L 3G1 glabahn@uwaterloo.ca 
The new coordinates (1) are also left invariant by the above transformation. We shall prove that they have the rather strong property that any dynamical system that is invariant under the transformations (2) can be written in terms of the variables (1) with the following substitution:

$$
r \mapsto 1, h \mapsto 1, K \mapsto 1, s \mapsto \mathfrak{s}, k \mapsto \mathfrak{k}, d \mapsto \mathfrak{d}, t \mapsto \mathfrak{t}, n \mapsto \mathfrak{n}, p \mapsto \mathfrak{p}
$$

Furthermore we shall show how to retrieve the solutions of the original system from the solutions of the reduced system. Here, if $(\mathfrak{n}(\mathfrak{t}), \mathfrak{p}(\mathfrak{t}))$ is a solution of the reduced system for the parameters $(\mathfrak{s}, \mathfrak{h}, \mathfrak{k})$ then, for any constant $(r, h, K)$, we obtain a solution of the original system with parameter $(r, h, K, s, k, d)$ by forming the following combinations:

$$
s=r \mathfrak{s}, k=r h \mathfrak{k}, d=K \mathfrak{d}, \quad n(t)=K \mathfrak{n}(r t), p(t)=\frac{K}{h} \mathfrak{p}(r t) .
$$

Note that the relationships (1-4) are all given by monomial maps, where we have allowed negative powers. As such they are appropriately described by matrices of integers. For instance the transformations (2) is described by a $3 \times 9$ matrix. If we use the order $(\eta, \mu, \nu)$ for the parameters of the transformations and $(r, K, k, s, h, d, t, n, p)$ for the variables of the system this matrix is

$$
A=\left[\begin{array}{rrrrrrrrr}
-1 & 0 & 0 & -1 & -1 & 0 & 1 & 0 & 0 \\
0 & 1 & 1 & 0 & 1 & 1 & 0 & 1 & 0 \\
0 & -1 & 0 & 0 & -1 & 0 & 0 & 0 & 1
\end{array}\right]
$$

In this paper we show how parameter reduction, as in the above example, can be algorithmically performed with linear algebra over the integers. This applies to a great number of models from mathematical biology.

Parameter reduction is actually a particular case of a more general problem to which we give a complete solution. We provide an algorithmic solution to scaling symmetry reduction of a dynamical system: determine a maximal scaling symmetry without isotropy, compute a generating set of rational invariants that act as new variables, obtain the dynamics on those new variables and finally establish the correspondence between the solutions of that reduced system and the original system. All those steps, except actually solving the differential systems, are algorithmic and relie solely on linear algebra over the integers.

Scalings form a class of group actions; They describe groups of transformation like (2) that rescales each individual variable. On the theoretical front scalings are known as torus actions and play a major role in algebraic geometry and combinatorics. Scalings also underlie what is known as dimensional analysis with the invariants giving the dimensionless quantities needed to derive physical laws [3, 4, 12]. Dimensional analysis has been automated in the works [13] and [15]. Central to this is the Buckingham- $\pi$-theorem. A reinterpretation of it states that a fundamental set of invariants is obtained from the basis of the nullspace of the matrix of exponents of the scaling [21, Section 3.4]. As illustrated in the above example scalings also give mathematical sense to rules of thumb applied to reduce the number of parameters in biological and physical models $[20,17]$. In this context, reduction by a scaling symmetry of a dynamical was previously studied with an algorithmic point of view in $[11,16,24]$. In this paper we go further in this direction than handled in the previous cited works.

Determining symmetries of differential equations has had many applications [21]. One usually resorts to infinitesimal methods and obtain local symmetries. When dealing with a dynamical system given in terms of rational functions, we determine the maximal scaling symmetry as a lattice kernel of an integer matrix. The group action thus determined is rational while retaining trivial isotropy.

Algorithmic tools for finding generating rational invariants and rewrite rules for the general class of rational actions of an algebraic group typically require Gröbner bases computations [9, 14, 19]. A rewriting substitution can be achieved provided we allow algebraic functions [10]. Gröbner bases are unnecessary for scalings; linear algebra over the integers provides more information. The key is to compute a Hermite normal form of the matrix describing the scaling. The information is then read on the unimodular multiplier giving the Hermite normal form, and its inverse. The unimodular multiplier provides a minimal generating 
set of rational invariants and the equations of a rational section. Its inverse provides the substitution to be performed to rewrite any invariant in terms of the exhibited generating set. When comparing to $[6,9,10,18]$ where the local cross-section has to be part of the input, an important point here is that a rational section, that is, a global cross-section (of degree 1), is actually a side-product of the algorithm.

As illustrated earlier, invariants can be used as new variables to simplify dynamical systems with a symmetry. When this symmetry is a scaling we show that the reduced dynamical system can be directly determined from the unimodular multiplier and its inverse. The relationship between the solutions of the reduced system and the original system can also be written down explicitly from those two matrices. The solutions of the original system are obtained from the solutions of the reduced system by additional mutually independent quadratures. This is to be compared and contrasted with the general methods of symmetry reduction proposed in $[1,7]$ and $[18$, Section 6]. Providing a global cross-section, and quotient map, does not make them straightforwardly algorithmic.

The unimodular multipliers for the Hermite normal form of the scaling matrices are not unique. We propose a normal form that exhibits further properties of the scaling. In particular, this normal form discriminates the cases where the scaling symmetry can be fully used to reduce the number of parameters in a dynamical system. The solutions of the original system are then obtained from the solution of the reduced system with just some additional constants.

The remainder of the paper is organized as follows. Section 2 presents the needed material on integer matrices and the Hermite normal form, along with the normalization of its unimodular multipliers. Section 3 presents scalings together with the matrix notations for monomial maps. Section 4 shows how to produce the generating invariants, rewrite rules and a rational section for a scaling. Section 5 provides an algorithm to compute the maximal scaling that leaves a given set of rational function invariants. The determination of the maximal scaling symmetry of a dynamical system is reduced to this problem. The scaling symmetry reduction of dynamical systems are discussed in Section 6. Section 7 shows how this can be specialized to explicitly reduce the number of parameters in dynamical systems, as mentioned earlier in the introduction.

\section{$2 \quad$ Integer Matrix Normal Forms}

When dealing with matrices of integers such basic operations as Gaussian elimination or finding a row echelon form are no longer valid since this involves working over the field of rational numbers. In this section we provide the basic information about the Hermite normal form of a matrix of integers, a type of triangularization for integer matrices. Here row and column operations are represented by unimodular matrices, which are invertible integer matrices whose inverses are also integer matrices. The unimodular multiplier to obtain the Hermite normal form of an integer matrix is not unique. We propose a normal form for it, which is relevant for our applications.

\subsection{Hermite Normal Forms}

Definition 2.1 An $m \times n$ integer matrix $H=\left[h_{i j}\right]$ is in column Hermite Normal Form if there exists an integer $r$ and a strictly increasing sequence $i_{1}<i_{2}<\cdots<i_{r}$ of pivot rows such that

(i) The first $r$ columns are nonzero;

(ii) $h_{k, j}=0$ for $k>i_{j}$;

(iii) $0 \leq h_{i_{j}, k}<h_{i_{j}, j}$ when $j<k$.

Thus a matrix is in column Hermite normal form if the submatrix formed by the pivot rows $i_{1}, \cdots, i_{r}$ and the first $r$ columns is upper triangular and that all nonzero elements of the pivot rows are positive and 
less than the corresponding (positive) diagonal entry. The integer $r$ is the rank of the matrix. By changing column to row and row and column indices in (ii) and (iii) one obtains the row Hermite Normal Form of a matrix of integers.

Every integer matrix can be transformed via integer column operations to obtain a unique column Hermite form. The column operations are encoded in unimodular matrices, that is, invertible integer matrices whose inverses are also integer matrices. Thus for each $A$ there exists a unimodular matrix $V$ such that $A \cdot V$ is in Hermite normal form. Similar statements also holds for the row Hermite normal form. We refer the reader to $[5,23]$ for more information on such forms.

When $A \in \mathbb{Z}^{r \times n}$, with $r \leq n$, has full row rank $r$ then there exists a unimodular matrix $V$ such that

$$
A \cdot V=[H, 0] \text { with } H \in \mathbb{Z}^{r \times r} \text { of full rank. }
$$

If $W \in \mathbb{Z}^{n \times n}$ is the inverse of $V$ then we can partition $V$ and $W$ as

$$
V=\left[V_{\mathfrak{i}}, V_{\mathfrak{n}}\right] \text { with } V_{\mathfrak{i}} \in \mathbb{Z}^{n \times r} \text { and } V_{\mathfrak{n}} \in \mathbb{Z}^{n \times(n-r)}
$$

and

$$
W=\left[\begin{array}{l}
W_{\mathfrak{u}} \\
W_{\mathfrak{d}}
\end{array}\right] \text { with } W_{\mathfrak{u}} \in \mathbb{Z}^{r \times n} \text { and } W_{\mathfrak{o}} \in \mathbb{Z}^{(n-r) \times n}
$$

We then have

$$
\begin{aligned}
& I_{n}=W V=\left[\begin{array}{ll}
W_{\mathfrak{u}} V_{\mathfrak{i}} & W_{\mathfrak{u}} V_{\mathfrak{n}} \\
W_{\mathfrak{d}} V_{\mathfrak{i}} & W_{\mathfrak{d}} V_{\mathfrak{n}}
\end{array}\right] \\
& I_{n}=V W=V_{\mathfrak{i}} W_{\mathfrak{u}}+V_{\mathfrak{n}} W_{\mathfrak{d}} .
\end{aligned}
$$

Note that the blocks of $V$ provide the column Hermite normal forms of the blocks of $W$ since from (8) we have

$$
W_{\mathfrak{u}} \cdot\left[V_{\mathfrak{i}}, V_{\mathfrak{n}}\right]=\left[I_{r}, 0\right] \quad \text { and } \quad W_{\mathfrak{d}} \cdot\left[V_{\mathfrak{n}}, V_{\mathfrak{i}}\right]=\left[I_{n-r}, 0\right] .
$$

We state a known property of Hermite normal forms $[5,23]$ in a way that is needed later in the paper.

Lemma 2.2 Let $A \in \mathbb{Z}^{r \times n}$ be a full row rank matrix and $V \in \mathbb{Z}^{n \times n}$ a unimodular matrix such that $A V=$ $[H, 0]$ with $H \in \mathbb{Z}^{r \times r}$. If $V$ is partitioned as in (6), then the columns of $V_{\mathfrak{n}}$ form a basis for the integer lattice defined by the kernel of $A$.

\section{$2.2 \quad$ Normal unimodular multiplier}

For the problem of interest in this paper the number of columns is larger than the rank. In this case the unimodular multiplier is not unique. Indeed, with the partition $V=\left[V_{\mathfrak{i}}, V_{\mathfrak{n}}\right]$ as in (6), we see that any column operations using the columns of $V_{\mathfrak{n}}$ do not affect the Hermite form $H$ for the initial matrix $A$ and hence results in a different unimodular multiplier $V$. In this subsection we describe a normalization of the multiplier $V$ which is both simple and unique. The normalization will play an important role in later sections.

Previous work on determining unique unimodular multipliers includes that of $[5,8]$ for integer matrices where the unimodular multiplier is reduced via lattice reduction. In our case we prefer that the component $V_{n}$ be in Hermite normal form as the triangular aspect of that form is useful later in our applications. Our construction is similar to that used for polynomial matrices in [2].

Theorem 2.3 Let $A \in \mathbb{Z}^{r \times n}$ be a full row rank matrix and $V \in \mathbb{Z}^{n \times n}$ a unimodular matrix such that $A V=[H, 0]$ with $H \in \mathbb{Z}^{r \times r}$ in column Hermite normal form. 
(i) A unimodular multiplier $V$ is unique up to multiplication on the right by matrices of the form

$$
\left[\begin{array}{cc}
I_{r} & 0 \\
M & U
\end{array}\right], \quad \text { with } U \in \mathbb{Z}^{(n-r) \times(n-r)} \quad \text { unimodular } .
$$

(ii) There exists a unique multiplier $V=\left[V_{\mathfrak{i}}, V_{\mathfrak{n}}\right]$ with

(a) $V_{\mathfrak{n}} \in \mathbb{Z}^{n \times(n-r)}$ in column Hermite normal form,

(b) If $i_{1}<i_{2}<\cdots<i_{n-r}$ are the pivot rows for $V_{n}$ then for each $1 \leq j \leq n-r$ :

$$
0 \leq\left[V_{\mathfrak{i}}\right]_{i_{j}, k}<\left[V_{\mathfrak{n}}\right]_{i_{j}, j} \text { for all } 1 \leq k \leq r .
$$

That is, $V_{\mathfrak{i}}$ is reduced with respect to the pivots rows of $V_{\mathfrak{n}}$.

PROOF: Suppose that $V^{(1)}=\left[V_{\mathfrak{i}}^{(1)}, V_{\mathfrak{n}}^{(1)}\right]$ and $V^{(2)}=\left[V_{\mathfrak{i}}^{(2)}, V_{\mathfrak{n}}^{(2)}\right]$ are two unimodular multipliers for the Hermite form which we both partition in the usual way. Then $V_{\mathfrak{n}}^{(1)}$ and $V_{\mathfrak{n}}^{(2)}$ are two bases for the nullspace of $A$ as a module over $\mathbb{Z}$. Thus there exists a unimodular matrix $U \in \mathbb{Z}^{(n-r) \times(n-r)}$ which makes these matrices column equivalent, that is, $V_{\mathfrak{n}}^{(1)}=V_{\mathfrak{n}}^{(2)} U$. Also, by the uniqueness of $H$, the columns of $V_{i}^{(1)}-V_{i}^{(2)}$ are in the nullspace of $A$ and hence there exists a matrix $M \in \mathbb{Z}^{(n-r) \times r}$ such that $V_{\mathfrak{i}}^{(1)}-V_{\mathfrak{i}}^{(2)}=V_{\mathfrak{n}}^{(2)} M$. This gives the general form of the multipliers in (i).

The existence of a unimodular multiplier $V$ satisfying (a) follows from the existence of column Hermite forms. The reduction (b) follows by doing the column operation

$$
\left[V_{\mathbf{i}}\right]_{i_{j}, k} \leftarrow\left[V_{\mathbf{i}}\right]_{i_{j}, k}-q \cdot\left[V_{\mathfrak{n}}\right]_{i_{j}, j} \quad \text { with } \quad q=\operatorname{iquo}\left(\left[V_{\mathbf{i}}\right]_{i_{j}, k},\left[V_{n}\right]_{i_{j}, j}\right)
$$

for each $k$ as $j$ varies from column $n-r$ to 1 . Here iquo denotes integer quotient, a function which always results in a nonnegative remainder.

It remains to show that any $V$ satisfying (ii) is unique. Thus we suppose the contrary and assume that we have $A \cdot V=A \cdot V^{*}=[H, 0]$ with $V=\left[V_{\mathfrak{i}}, V_{\mathfrak{n}}\right]$ and $V^{*}=\left[V_{\mathrm{i}}^{*}, V_{\mathfrak{n}}^{*}\right]$ both being unimodular and satisfying (ii). From (i) we have that there exists an integer matrix $U$ such that $V_{\mathfrak{n}}=V_{\mathfrak{n}}^{*} \cdot U$. The uniqueness of column Hermite forms then implies that $U=I$ and so $V_{\mathfrak{n}}=V_{\mathfrak{n}}^{*}$.

Suppose now that $M \in \mathbb{Z}^{(n-r) \times r}$ such that $V_{\mathfrak{i}}-V_{i}^{*}=V_{\mathfrak{n}} \cdot M$. Looking at the last pivot row of $V_{\mathfrak{n}}$ (row $i_{n-r}$ ) and using condition (b) we have that for each $1 \leq k \leq r$ :

$$
\left[V_{\mathrm{i}}\right]_{i_{n-r}, k}-\left[V_{\mathrm{i}}^{*}\right]_{i_{n-r}, k}=\left[V_{\mathfrak{n}}\right]_{i_{n-r}, n-r} \cdot[M]_{n-r, k} .
$$

From condition (b) we have that both $\left[V_{\mathfrak{i}}\right]_{i_{n-r}, k}$ and $\left[V_{\mathfrak{i}}^{*}\right]_{i_{n-r}, k}$ are positive integers smaller than $\left[V_{\mathfrak{n}}\right]_{i_{n-r}, n-r}$. Thus $[M]_{n-r, k}=0$ for all $k$ and hence the last row of $M$ is zero. Suppose now that rows $n-r, \ldots, \ell+1$ of $M$ are all zero. Then for the pivot row $i_{\ell}$ the triangular property of the Hermite form implies that for each $k$ we have

$$
\left[V_{\mathfrak{i}}\right]_{i_{\ell}, k}-\left[V_{\mathfrak{i}}^{*}\right]_{i_{\ell}, k}=\left[V_{\mathfrak{n}}\right]_{i_{\ell}, \ell} \cdot[M]_{\ell, k} .
$$

As before, the size condition (c) implies that $[M]_{\ell, k}=0$ for all $k$ and hence row $\ell$ of $M$ is zero. By induction we see that $M=0$. Hence $V_{\mathrm{i}}=V_{\mathrm{i}}^{*}$ and so $V=V^{*}$ is unique.

We refer to our unique unimodular multiplier $V$ as a normal unimodular multiplier. The construction of this multiplier is similar to that of [5, page 97] except that both $H$ and $V_{\mathfrak{n}}$ are lattice reduced in that case.

Example 2.4 Let

$$
A=\left[\begin{array}{ccccc}
8 & 2 & 15 & 9 & 11 \\
6 & 0 & 6 & 2 & 3
\end{array}\right]
$$


which has Hermite normal form $\left[I_{2}, 0\right]$. The reduction performed by Maple results in the unimodular multiplier

$$
V^{\prime}=\left[\begin{array}{rrrrr}
-49 & -1 & -57 & -13 & -28 \\
-36 & -1 & -42 & -10 & -21 \\
79 & 2 & 92 & 21 & 45 \\
-36 & -1 & -42 & -9 & -21 \\
-36 & -1 & -42 & -10 & -20
\end{array}\right]
$$

while the normalized unimodular multiplier is

$$
V=\left[\begin{array}{rrrrr}
-1 & -2 & -2 & -2 & -1 \\
-3 & -14 & -7 & -13 & -7 \\
1 & 1 & 2 & 1 & 0 \\
0 & 2 & 0 & 3 & 0 \\
0 & 1 & 0 & 0 & 2
\end{array}\right]
$$

The simplest case of a normal unimodular multiplier occurs when the pivot rows of $V_{\mathfrak{n}} \in \mathbb{Z}^{n \times(n-r)}$ are the rows of an $(n-r)$-identity matrix. In this case there we have

$$
V_{\mathfrak{n}}=\left[\begin{array}{c}
V_{\mathfrak{n}}^{*} \\
I_{n-r}
\end{array}\right] \text { and } V_{\mathfrak{i}}=\left[\begin{array}{c}
V_{\mathfrak{i}}^{*} \\
0_{r}
\end{array}\right] \text {. }
$$

Thus $V_{\mathrm{i}}^{*}$ is unimodular and $V$ and $W\left(\right.$ where $W=V^{-1}$ ) are of the form

$$
V=\left[\begin{array}{cc}
V_{\mathrm{i}}^{*} & V_{\mathfrak{n}}^{*} \\
0 & I_{n-r}
\end{array}\right] \text { and } W=\left[\begin{array}{cc}
V_{\mathfrak{i}}^{*-1} & -V_{\mathfrak{i}}^{*-1} V_{\mathfrak{n}}^{*} \\
0 & I_{n-r}
\end{array}\right] .
$$

Note that this is the only possibility for a block of zero to appear at the bottom left. Indeed, if $V$ is block upper triangular, the diagonal blocks need to be unimodular and the Hermite normal form of a unimodular matrix is the identity.

\subsection{Reducing the unimodular inverse}

For some applications it is meaningful to rather reduce or simplify the inverse $W$ of a unimodular multiplier for the Hermite form. This latter is defined by the fact that it is unimodular and satisfies

$$
A=[H, 0] \cdot W .
$$

By Theorem 2.3, if $W$ is the inverse of a unimodular multiplier $V$ then any other such inverse is of the form

$$
\left[\begin{array}{cc}
I_{r} & 0 \\
M^{\prime} & U^{\prime}
\end{array}\right] \cdot W \quad \text { with } U^{\prime} \in \mathbb{Z}^{(n-r) \times(n-r)} \quad \text { unimodular. }
$$

Thus if we partition any $W$ satisfying $(9)$ as $\left[\begin{array}{l}W_{\mathfrak{u}} \\ W_{\mathfrak{o}}\end{array}\right]$ then $W_{\mathfrak{u}}$ is unique and any simplification can only take place with $W_{\mathfrak{o}}$.

An obvious simplification to consider is the row Hermite form of $W_{\mathfrak{d}}$, that is, $M^{\prime}=0$ and $U^{\prime}$ such that $U^{\prime} W_{\mathfrak{d}}=W_{\mathfrak{d}}^{*}$, the row Hermite form of $W_{\mathfrak{d}}$. When applied to the inverse of the normal unimodular multiplier we call the result the reduced unimodular inverse.

Example 2.5 Continuing with Example 2.4, a unimodular inverse $W$ for the normal unimodular multiplier $V$ is

$$
W=\left[\begin{array}{l}
W_{\mathfrak{u}} \\
W_{\mathfrak{o}}
\end{array}\right]=\left[\begin{array}{rrrrr}
8 & 2 & 15 & 9 & 11 \\
6 & 0 & 6 & 2 & 3 \\
\hline-5 & -1 & -8 & -5 & -6 \\
-4 & 0 & -4 & -1 & -2 \\
-3 & 0 & -3 & -1 & -1
\end{array}\right]
$$


Taking the row Hermite normal form $W_{\mathfrak{d}}^{*}$ of $W_{\mathfrak{o}}$ then gives a reduced inverse multiplier and corresponding unimodular multiplier $V^{*}$ as

$$
W^{*}=\left[\begin{array}{l}
W_{\mathfrak{u}} \\
W_{\mathfrak{d}}^{*}
\end{array}\right]=\left[\begin{array}{rrrrr}
8 & 2 & 15 & 9 & 11 \\
6 & 0 & 6 & 2 & 3 \\
\hline 1 & 0 & 1 & 0 & 1 \\
0 & 1 & 3 & 0 & 11 \\
0 & 0 & 0 & 1 & -2
\end{array}\right] \text { and } V^{*}=\left[\begin{array}{cc|ccc}
-1 & -2 & 21 & 2 & 13 \\
-3 & -14 & 108 & 7 & 55 \\
1 & 1 & -14 & -2 & -11 \\
0 & 2 & -12 & 0 & -3 \\
0 & 1 & -6 & 0 & -2
\end{array}\right] \text {. }
$$

The reduction we have defined for the inverse $W$ might fail to provide a normal form. If a normal form is needed then it can be obtained as follows. Since $W_{\mathfrak{u}}$ has full rank one can always first reduce $W_{\mathfrak{d}}$ with respect to $W_{\mathfrak{u}}$ and then reduce the resulting $W_{\mathfrak{o}}$. If $W_{\mathfrak{u}}^{*}$ is the row Hermite form for $W_{\mathfrak{u}}$ then this is the same as first finding $M \in \mathbb{Z}^{n-r \times r}$ such that $M W_{\mathfrak{u}}^{*}+W_{\mathfrak{d}}=W_{\mathfrak{d}}^{*}$ where the entries of $W_{\mathfrak{d}}^{*}$ are smaller than $W_{\mathfrak{u}}^{*}$ and then finding $U \in \mathbb{Z}^{(n-r) \times(n-r)}$ so that $U W_{\mathfrak{d}}^{*}$ is in row Hermite form. Here smaller means that $0 \leq\left[W_{\mathfrak{d}}^{*}\right]_{k, c_{i}}<\left[W_{\mathfrak{u}}^{*}\right]_{i, c_{i}}$ for all $k$ and $i$ where the $c_{i}$ are the pivot columns for $W_{\mathfrak{u}}^{*}$.

\section{Scalings}

Scalings can be described through the matrix of exponents of the group parameters as they act on each component ${ }^{1}$. In this section we describe the matrix forms and properties that are useful when representing scalings and computing their invariants. We consider an algebraically closed field $\mathbb{K}$ of characteristic zero, the multiplicative group of which is $\mathbb{K}^{*}$.

\subsection{Matrix notations for monomial maps}

If $a=\left[a_{1}, \ldots, a_{r}\right]^{T}$ is a column vector of integers and $\lambda=\left[\lambda_{1}, \ldots, \lambda_{r}\right]$ is a row vector with entries in $\mathbb{K}^{*}$, then $\lambda^{a}$ denotes the scalar

$$
\lambda^{a}=\lambda_{1}^{a_{1}} \cdots \lambda_{r}^{a_{r}} .
$$

If $\lambda=\left[\lambda_{1}, \ldots, \lambda_{r}\right]$ is a row vector of $r$ indeterminates, then $\lambda^{a}$ can be understood as a monomial in the Laurent polynomial ring $\mathbb{K}\left[\lambda, \lambda^{-1}\right]$, a domain isomorphic to $\mathbb{K}[\lambda, \mu] /\left(\lambda_{1} \mu_{1}-1, \ldots, \lambda_{r} \mu_{r}-1\right)$. We extend this notation to matrices: If $A$ is an $r \times n$ matrix then $\lambda^{A}$ is the row vector

$$
\lambda^{A}=\left[\lambda^{A_{,, 1}}, \cdots, \lambda^{A, n}\right]
$$

where $A_{\cdot, 1}, \ldots, A_{,, n}$ are the $n$ columns of $A$.

In some cases it is important to keep track of those exponents which are nonnegative (and hence describe numerators) and those which are negative (and hence describe denominators). To this end the following notation becomes useful. Every vector $a \in \mathbb{Z}^{r}$ can be uniquely written as $a=a^{+}-a^{-}$where $a^{+}$and $a^{-}$are nonnegative and have disjoint support. Their components are:

$$
\left[a^{+}\right]_{i}=\left\{\begin{array}{cc}
a_{i} & \text { if } a_{i} \geq 0 \\
0 & \text { otherwise }
\end{array} \text { and }\left[a^{-}\right]_{i}=\left\{\begin{array}{cc}
a_{i} & \text { if } a_{i} \leq 0 \\
0 & \text { otherwise }
\end{array}\right.\right.
$$

This can be extended to $r \times n$ matrices by

$$
A^{+}=\left[\left(A_{\cdot, 1}\right)^{+}, \cdots,\left(A_{\cdot, n}\right)^{+}\right] \text {and } A^{-}=\left[\left(A_{\cdot, 1}\right)^{-}, \cdots,\left(A_{\cdot, n}\right)^{-}\right] .
$$

If $x=\left[x_{1}, \ldots, x_{n}\right]$ and $y=\left[y_{1}, \ldots, y_{n}\right]$ are two row vectors, we write $x \star y$ for the row vector obtained by component wise multiplication:

$$
x \star y=\left[x_{1} y_{1}, \ldots, x_{n} y_{n}\right]
$$

\footnotetext{
${ }^{1}$ Similar descriptions are used for the parameterization of toric varieties [25].
} 
Proposition 3.1 Suppose $A$ and $B$ are matrices of size $r \times n$ and $n \times n$, respectively, and that $\lambda$ is a row vector with $r$ components. Then

(a) If $A=\left[A_{\mathfrak{i}}, A_{\mathfrak{n}}\right]$ is a partition of the columns of $A$, then $\lambda^{A}=\left[\lambda^{A_{\mathfrak{i}}}, \lambda^{A_{\mathfrak{n}}}\right]$,

(b) $\lambda^{A B}=\left(\lambda^{A}\right)^{B}$,

Suppose $A$ and $B$ are matrices of size $r \times n$ and $\lambda$ and $\mu$ are row vectors with $r$ components. Then

(c) $(\lambda \star \mu)^{A}=\lambda^{A} \star \mu^{A}$.

(d) $\lambda^{A+B}=\lambda^{A} \star \lambda^{B}$

PROOF: Part (a) follows directly from the definition of $\lambda^{A}$. For part (b) we have for each component $j$, $1 \leq j \leq t$ :

$$
\begin{aligned}
{\left[\left(\lambda^{A}\right)^{B}\right]_{j} } & =\prod_{i=1}^{n}\left[\lambda^{A}\right]_{i}^{b_{i j}} \\
& =\prod_{i=1}^{n}\left(\prod_{\ell=1}^{r} \lambda_{\ell}^{a_{\ell i}}\right)^{b_{i j}} \\
& =\prod_{\ell=1}^{r}\left(\prod_{i=1}^{n} \lambda_{\ell}^{a_{\ell i} b_{i j}}\right)=\prod_{\ell=1}^{r}\left(\lambda_{\ell}^{\sum_{i=1}^{n} a_{\ell i} b_{i j}}\right)=\left[\lambda^{A B}\right]_{j} .
\end{aligned}
$$

For part (c) one simply notices that for each $j$ we have

$$
\begin{aligned}
{\left[(\lambda \star \mu)^{A}\right]_{j} } & =\prod_{i}^{r}[\lambda \star \mu]_{i}^{a_{i, j}}=\prod_{i}^{r} \lambda_{i}^{a_{i, j}} \cdot \mu_{i}^{a_{i, j}} \\
& =\left[\lambda^{A}\right]_{j}\left[\mu^{A}\right]_{j}=\left[\lambda^{A} \star \mu^{A}\right]_{j} .
\end{aligned}
$$

The proof of (d) follows along the same lines.

\subsection{Scalings in matrix notation}

The $r$-dimensional torus is the Abelian group $\left(\mathbb{K}^{*}\right)^{r}$. Its identity is $1_{r}=(1, \ldots, 1)$ and the group operation is component-wise multiplication, which we denoted $\star$.

Definition 3.2 Let $A$ be a $r \times n$ integer matrix: $A \in \mathbb{Z}^{r \times n}$. The associated scaling is the linear action of $\left(\mathbb{K}^{*}\right)^{r}$ on the affine space $\mathbb{K}^{n}$ given by

$$
\begin{aligned}
\left(\mathbb{K}^{*}\right)^{r} \times \mathbb{K}^{n} & \rightarrow \mathbb{K}^{n} \\
(\lambda, \quad z) & \rightarrow \lambda^{A} \star z .
\end{aligned}
$$

With the notations introduced above we have that

$$
\lambda^{A} \star z=\left[\lambda^{A \cdot, 1} z_{1}, \ldots, \lambda^{A,, n} z_{n}\right]
$$

with $A_{\cdot, 1}, \ldots, A_{\cdot, n}$ being the $n$ columns of $A$. Thus for each $j=1, \ldots, n$ the action scales the $j^{\text {th }}$ component $z_{j}$ by the power product $\lambda_{1}^{a_{1, j}} \cdots \lambda_{r}^{a_{r, j}}$. The axioms for a group action are satisfied thanks to Proposition 3.1: $1_{r} \star z=z$ and $(\lambda \star \mu)^{A} \star z=\lambda^{A} \star\left(\mu^{A} \star z\right)$.

There is no loss of generality in assuming that $A$ has full row rank. Indeed, we can view the scaling defined by $A$ as a diagonal representation of $\left(\mathbb{K}^{*}\right)^{r}$ on the $n$ dimensional space $\mathbb{K}^{n}$ :

$$
\begin{array}{ccc}
\left(\mathbb{K}^{*}\right)^{r} & \rightarrow & \mathrm{D}_{n} \\
\left(\lambda_{1}, \ldots, \lambda_{r}\right) & \mapsto \operatorname{diag}\left(\lambda^{A}\right)
\end{array}
$$


where $\mathrm{D}_{n}$ is the group of invertible diagonal matrices. This in turn can be factored by the group morphism from $\left(\mathbb{K}^{*}\right)^{r}$ to $\left(\mathbb{K}^{*}\right)^{n}$ defined by $A$. This is given explicitly by:

$$
\begin{aligned}
& \rho(A): \quad\left(\mathbb{K}^{*}\right)^{r} \quad \rightarrow \quad\left(\mathbb{K}^{*}\right)^{n} \\
& \left(\lambda_{1}, \ldots, \lambda_{r}\right) \mapsto \quad \lambda^{A}
\end{aligned}
$$

Suppose now that $U A=\left[\begin{array}{l}B \\ 0\end{array}\right]$ is in row Hermite normal form ${ }^{2}$, with unimodular row multiplier $U$. Consider the splitting $U=\left[\begin{array}{l}U_{1} \\ U_{2}\end{array}\right]$, where $U_{1} A=B$ is of row dimension $d$ and $U_{2} A=0$. Then

$$
\begin{array}{ccccc}
\left(\mathbb{K}^{*}\right)^{d} \times\left(\mathbb{K}^{*}\right)^{r-d} & \stackrel{U}{\rightarrow} & \left(\mathbb{K}^{*}\right)^{r} & \stackrel{A}{\rightarrow} & \left(\mathbb{K}^{*}\right)^{n} \\
\left(\mu_{1}, \mu_{2}\right) & \mapsto & \mu_{1}^{U_{1}} \star \mu_{2}^{U_{2}} & \mapsto & \left(\mu_{1}^{U_{1}} \star \mu_{2}^{U_{2}}\right)^{A}=\mu_{1}^{B} .
\end{array}
$$

Since $U$ is unimodular, $\rho(U)$ is an isomorphism of groups and the image of $\left(\mathbb{K}^{*}\right)^{r}$ by $\rho(A)$ is equal to the image of $\left(\mathbb{K}^{*}\right)^{d}$ by $\rho(B)$.

Proposition 3.3 Let $A$ be a full row rank matrix in $\mathbb{Z}^{r \times n}$. The isotropy groups for the scaling defined by $A$ on $\left(\mathbb{K}^{*}\right)^{n}$ are trivial if and only if the Hermite normal form of $A$ is $\left[I_{r}, 0\right]$.

PROOF: Assume $V=\left[V_{\mathfrak{i}}, V_{\mathfrak{n}}\right]$ is a unimodular multiplier such that $A \cdot V$ is in Hermite normal form $[H, 0]$. Take $z \in\left(\mathbb{K}^{*}\right)^{n}$ so that there exists $\lambda \in\left(\mathbb{K}^{*}\right)^{r}$ such that $\lambda^{A} \star z=z$. This is equivalent to $\left[\lambda^{H} \star z^{V_{\mathrm{i}}}, z^{V_{\mathrm{n}}}\right]=z^{V}$ and therefore to $\lambda^{H}=1_{r}$.

Since $H$ is triangular with positive integer entries on the diagonal, the set of equations $\lambda^{H}=1_{r}$ has $\prod_{i=1}^{r} h_{i}$ distinct solutions, where $\left(h_{i}\right)$ are the diagonal entries. In all cases, $\lambda=1_{r}$ is a solution. It is the only solution if and only if $H=I_{r}$.

\section{Rational invariants of scaling}

Consider a full row rank matrix $A \in \mathbb{Z}^{r \times n}$ which defines a scaling, that is an action of the torus $\left(\mathbb{K}^{*}\right)^{r}$ on $\mathbb{K}^{n}$. A rational invariant is an element $f$ of $\mathbb{K}(z)$ such that $f\left(\lambda^{A} \star z\right)=f(z)$. Rational invariants form the subfield $\mathbb{K}(z)^{A}$ of $\mathbb{K}(z)$. In this section we show how a unimodular multiplier $V$, where $A \cdot V$ is in Hermite normal form, provides us with a complete description of the subfield of rational invariants. From $V$, and its inverse, we shall extract

- $n-r$ generating rational invariants that are algebraically (and functionally) independent

- a simple rewriting of any (rational) invariant in terms of this generating set,

- a rational section to the orbits of the scaling.

We thus go much further than the group action transcription of the Buckingham $\pi$-theorem of dimensional analysis [3,21]. This latter takes any basis of the nullspace of the matrix $A$ and provides a set of functionally generating invariants, some of which could involve fractional powers. In the present approach, only integer powers are involved. This spares us the determination of proper domains of definition. Furthermore, the Buckingham $\pi$-theorem gives no indication on how to rewrite an invariant in terms of the generators produced. The rewriting we propose is a simple substitution. This is reminiscent of the normalized invariants appearing

\footnotetext{
${ }^{2}$ Or any row rank revealing form.
} 
in $[6,10,18]$ (or replacement invariants in [9]). And rightfully so. Using the terminology of those articles, we are in a position to exhibit a global cross-section (of degree one) to the orbits of the scaling. Note though, that the substitution is again rational: we do not introduce any algebraic functions as would be generally the case when choosing a local cross-section arbitrarily.

\subsection{Generating and replacement invariants}

A Laurent monomial $z^{v}$ is a rational invariant if $\left(\lambda^{A} \star z\right)^{v}=z^{v}$ and therefore if and only if $A v=0$. The following theorem shows that rational invariants of a scaling can be written as a rational function of Laurent monomials that are invariants.

Lemma 4.1 Suppose $\frac{p}{q} \in \mathbb{K}(z)^{A}$, with $p, q \in \mathbb{K}[z]$ relatively prime. Then there exists $u \in \mathbb{Z}^{n}$ such that

$$
p(z)=\sum_{v \in \operatorname{ker} A \cap \mathbb{Z}^{n}} a_{v} z^{u+v} \text { and } q(z)=\sum_{v \in \operatorname{ker} A \cap \mathbb{Z}^{n}} b_{v} z^{u+v}
$$

where the families of coefficients, $\left(a_{v}\right)_{v}$ and $\left(b_{v}\right)_{v}$, have finite support..$^{3}$

PROOF: We take advantage of the more general fact that rational invariants of a linear action on $\mathbb{K}^{n}$ are quotients of semi-invariants (see for instance [22, Theorem 3.3]). Indeed, if $p / q$ is a rational invariant, then we have

$$
p(z) q\left(\lambda^{A} \star z\right)=p\left(\lambda^{A} \star z\right) q(z)
$$

in $\mathbb{K}(\lambda)[z]$. As $p$ and $q$ are relatively prime, $p(z)$ divides $p\left(\lambda^{A} \star z\right)$ and, since these two polynomials have the same degree, there exists $\chi(\lambda) \in \mathbb{K}(\lambda)$ such that $p\left(\lambda^{A} \star z\right)=\chi(\lambda) p(z)$. It then also follows that $q\left(\lambda^{A} \star z\right)=\chi(\lambda) q(z)$.

Let us now look at the specific case of a scaling. Then

$$
p(z)=\sum_{w \in \mathbb{Z}^{n}} a_{w} z^{w} \Rightarrow p\left(\lambda^{A} \star z\right)=\sum_{w \in \mathbb{Z}^{n}} a_{w} \lambda^{A w} z^{w} .
$$

For $p\left(\lambda^{A} \star z\right)$ to factor as $\chi(\lambda) p(z)$ we must have $A w=A u$ for any two vectors $u, w \in \mathbb{Z}^{n}$ with $a_{v}$ and $a_{u}$ in the support of $p$. Let us fix $u$. Then $w-u \in \operatorname{ker} A$ and $\chi(\lambda)=\lambda^{A u}$. From the previous paragraph we have $\sum_{w \in \mathbb{Z}^{n}} b_{w} \lambda^{A w} z^{w}=q\left(\lambda^{A} \star z\right)=\lambda^{A u} q(z)=\lambda^{A u} \sum_{w \in \mathbb{Z}^{n}} b_{w} z^{w}$. Thus $A u=A w$ and therefore there exists $v \in \operatorname{ker} A \cap \mathbb{Z}^{n}$ such that $w=u+v$ for all $w$ with $b_{w}$ in the support of $q$.

The set of rational functions on $\mathbb{K}^{n}$ that are invariant under a group action form a subfield of $\mathbb{K}(z)$ and, as such, it is a finitely generated field. In the case of a scaling the generators of this field can be constructed making use only of linear algebra and the representation of rational invariants given in Lemma 4.1.

Theorem 4.2 Let $V=\left[V_{\mathfrak{i}}, V_{\mathfrak{n}}\right]$ and $W=\left[\begin{array}{l}W_{\mathfrak{u}} \\ W_{\mathfrak{d}}\end{array}\right]$ be unimodular matrices of integers such that $A V=$ $[H, 0]$ is in column Hermite normal form and $W$ is the inverse of $V$. Then the scaling defined by $A$ has the following properties:

(a) The $n-r$ components of $g=\left[z_{1}, \ldots, z_{n}\right]^{V_{\mathfrak{n}}}$ form a generating set of rational invariants;

(b) Any rational invariant can be written in terms of the components of $g$ by substituting $z=\left[z_{1}, \ldots, z_{n}\right]$ by the respective components of $g^{W_{0}}$.

\footnotetext{
${ }^{3}$ In particular $a_{v}=0$ (respectively $b_{v}=0$ ) when $u+v \notin \mathbb{N}^{n}$.
} 
PROOF: Observe first that the components of $g$ are invariants. Indeed the columns of $V_{\mathfrak{n}} \operatorname{span} \operatorname{ker} A$ and so $\left(\lambda^{A} \star z\right)^{V_{\mathfrak{n}}}=\lambda^{A V_{\mathfrak{n}}} \star z^{V_{\mathfrak{n}}}=z^{V_{\mathfrak{n}}}$. We shall prove that any rational invariant can be rewritten in terms of these components.

Since $V$ and $W$ are inverses of each other we have $I_{n}=V_{\mathfrak{i}} W_{\mathfrak{u}}+V_{\mathfrak{n}} W_{\mathfrak{d}}$. Thus $z=z^{V_{\mathfrak{i}} W_{\mathfrak{u}}+V_{\mathfrak{n}} W_{\mathfrak{o}}}$, where $z=\left[z_{1}, \ldots, z_{n}\right]^{T}$, the vector of degree 1 monomials. More generally, for any $v \in \mathbb{Z}^{n}, z^{v}=z^{\left(V_{\mathfrak{i}} W_{\mathfrak{u}}+V_{\mathfrak{n}} W_{\mathfrak{\jmath}}\right) v}$. If now $v \in \operatorname{ker} A \cap \mathbb{Z}^{n}$ then $z^{v}=z^{V_{n} W_{\mathfrak{o}} v}=g^{W_{d} v}$ since ker $A \subset \operatorname{ker} W_{\mathfrak{u}}$.

The representation given in Lemma 4.1 implies that any $\frac{p}{q} \in \mathbb{K}(z)^{T}$, with $p, q \in \mathbb{K}[z]$ relatively prime, has the form

$$
p(z)=\sum_{v \in \operatorname{ker} A \cap \mathbb{Z}^{n}} a_{v} z^{u+v} \text { and } q(z)=\sum_{v \in \operatorname{ker} A \cap \mathbb{Z}^{n}} b_{v} z^{u+v}
$$

for some $u \in \mathbb{Z}^{n}$. As elements of $\mathbb{K}(z)$, we can rewrite these as

$$
p(z)=z^{u} \sum_{v \in \operatorname{ker} A \cap \mathbb{Z}^{n}} a_{v}\left(z^{V_{\mathfrak{n}} W_{\mathfrak{o}}}\right)^{v} \quad \text { and } \quad q(z)=z^{u} \sum_{v \in \operatorname{ker} A \cap \mathbb{Z}^{n}} b_{v}\left(z^{V_{\mathfrak{n}} W_{\mathfrak{o}}}\right)^{v}
$$

and so

$$
\frac{p(z)}{q(z)}=\frac{p\left(z^{V_{\mathfrak{n}} W_{\mathfrak{o}}}\right)}{q\left(z^{V_{\mathfrak{n}} W_{\mathfrak{o}}}\right)}=\frac{p\left(g^{W_{\mathfrak{o}}}\right)}{q\left(g^{W_{\mathfrak{o}}}\right)}
$$

Both $V$ and $W$ are needed for computing invariants and rewrite rules. Since a matrix $V$ is produced from column operations converting $A$ to Hermite normal form, the $W$ matrix can be computed simultaneously with minimal cost by the inverse column operations.

Example 4.3 Consider the scaling defined by $A=\left[\begin{array}{ll}2 & 3\end{array}\right]$. A unimodular multiplier for its Hermite normal form is

$$
V=\left[\begin{array}{rr}
-1 & 3 \\
1 & -2
\end{array}\right] \quad \text { with inverse } \quad W=\left[\begin{array}{ll}
2 & 3 \\
1 & 1
\end{array}\right]
$$

It follows that $g=\frac{x^{3}}{y^{2}}$ is a generating invariant. Any other rational invariant can be written in terms of $g$ with the substitution $x \mapsto g, y \mapsto g$.

Example 4.4 Consider the $2 \times 5$ matrix A given by

$$
A=\left[\begin{array}{rrrrr}
6 & 0 & -4 & 1 & 3 \\
0 & 3 & 1 & -4 & 3
\end{array}\right]
$$

If $z=\left(z_{1}, z_{2}, z_{3}, z_{4},, z_{5}\right)$ and $\lambda=(\mu, \nu)$ then the group action defined by $A$ is given by

$$
\lambda^{A} \star z=\left(\mu^{6} z_{1}, \nu^{3} z_{2}, \frac{\nu}{\mu^{4}} z_{3}, \frac{\mu}{\nu^{4}} z_{4}, \mu^{3} \nu^{3} z_{5}\right) .
$$

The column Hermite normal form for $A$ is given by

$$
\left[\begin{array}{ll}
H, & 0
\end{array}\right]=\left[\begin{array}{lllll}
3 & 2 & 0 & 0 & 0 \\
0 & 1 & 0 & 0 & 0
\end{array}\right]
$$

and the normal unimodular multiplier and its inverse are

$$
V=\left[\begin{array}{rr|rrr}
1 & 1 & 2 & 1 & 0 \\
1 & 0 & -1 & 2 & 0 \\
1 & 1 & 3 & 2 & 1 \\
1 & 0 & 0 & 2 & 1 \\
0 & 0 & 0 & 0 & 1
\end{array}\right], W=\left[\begin{array}{rrrrr}
2 & -2 & -2 & 3 & -1 \\
0 & 3 & 1 & -4 & 3 \\
\hline 0 & -1 & 0 & 1 & -1 \\
-1 & 1 & 1 & -1 & 0 \\
0 & 0 & 0 & 0 & 1
\end{array}\right]
$$


Here the last 3 rows of $V_{\mathfrak{n}}$ are the pivot rows. A generating set of invariants is given by the components

$$
\left(g_{1}, g_{2}, g_{3}\right)=z^{V_{\mathfrak{n}}}=\left(\frac{z_{1}^{2}}{z_{2}} z_{3}^{3}, \quad z_{1} z_{2}^{2} z_{3}^{2} z_{4}^{2}, \quad z_{3} z_{4} z_{5}\right)
$$

while the rewrite rules are given by

$$
\left(z_{1}, z_{2}, z_{3}, z_{4}, z_{5}\right) \rightarrow g^{W_{\mathfrak{o}}}=\left(\frac{1}{g_{2}}, \frac{g_{2}}{g_{1}}, g_{2}, \frac{g_{1}}{g_{2}}, \frac{g_{3}}{g_{1}}\right) .
$$

A normal unimodular multiplier $V=\left[V_{\mathfrak{i}}, V_{\mathfrak{n}}\right]$ has $V_{\mathfrak{n}}$ in column Hermite normal form. By part (a) of Theorem 4.2 the use of a normal unimodular multiplier implies a simple, triangular-like form for the invariants of a scaling. On the other hand a reduced unimodular inverse $W$ has $W_{\mathfrak{d}}$ in row Hermite normal form. By part (b) of Theorem 4.2 the use of a reduced unimodular inverse ensures a simple triangular-like form for the rewrite rules of an invariant of a scaling.

Example 4.5 Returning to the previous example the reduced inverse is

$$
W:=\left[\begin{array}{rrrrr}
2 & -2 & -2 & 3 & -1 \\
0 & 3 & 1 & -4 & 3 \\
\hline 1 & 0 & -1 & 0 & 0 \\
0 & 1 & 0 & -1 & 0 \\
0 & 0 & 0 & 0 & 1
\end{array}\right], \quad \text { with } \quad V=\left[\begin{array}{rr|rrr}
1 & 1 & -1 & -1 & -2 \\
1 & 0 & -2 & 3 & 1 \\
1 & 1 & -2 & -1 & -2 \\
1 & 0 & -2 & 2 & 1 \\
0 & 0 & 0 & 0 & 1
\end{array}\right] .
$$

Now the bottom 3 rows of $W$ are in row Hermite form. Accordingly the rewrite rules are given by

$$
\left(z_{1}, z_{2}, z_{3}, z_{4}, z_{5}\right) \rightarrow y^{W_{\mathfrak{o}}}=\left(g_{1}, g_{2}, \frac{1}{g_{1}}, \frac{1}{g_{2}}, g_{3}\right) .
$$

where the generating invariants, read from $V$, are

$$
\left(g_{1}, g_{2}, g_{3}\right)=z^{V_{\mathbf{n}}}=\left(\frac{1}{z_{1} z_{2}^{2} z_{3}^{2} z_{4}^{2}}, \frac{z_{2}^{3} z_{4}^{2}}{z_{1} z_{3}}, \frac{z_{2} z_{4} z_{5}}{z_{1}^{2} z_{3}^{2}}\right) .
$$

\subsection{Rational section to the orbits}

The fact that we can rewrite any invariant in terms of the generating set by a simple substitution actually reflects the existence and intrinsic use of a rational section [9, 10]. Indeed, any unimodular multiplier for the Hermite normal form provides a rational section. The simplest rational sections are uncovered by the normal unimodular multipliers of Theorem 2.3 .

An irreducible variety $\mathcal{P} \subset \mathbb{K}^{n}$ is a rational section for the rational action of an affine algebraic group if there exists a nonempty Zariski open subset $\mathcal{Z} \subset \mathbb{K}^{n}$ such that any orbit of the induced action on $\mathcal{Z}$ intersects $\mathcal{P}$ at exactly one point [22, Section 2.5].

Theorem 4.6 With the hypotheses of Theorem 4.2, the variety $\mathcal{P}$ of $\left(z^{V_{\mathrm{i}}^{+}}-z^{V_{\mathrm{i}}^{-}}\right): z^{\infty}$ is a rational section for the scaling defined by $A$. The intersection of the orbit of a point $z \in\left(\mathbb{K}^{*}\right)^{n}$ with this section is the point $z^{V_{\mathrm{n}} W_{\mathrm{o}}}$.

PRoOF: The matrix $W_{\mathfrak{o}}$ is full row rank and $W_{\mathfrak{d}} \cdot\left[V_{\mathfrak{n}}, V_{\mathfrak{i}}\right]=\left[I_{n-r}, 0\right]$. By Lemma 2.2 the columns of $V_{\mathfrak{i}}$ span the lattice kernel of $W_{\mathfrak{d}}$. Thus the kernel of

$$
\begin{array}{ccc}
\mathbb{K}[z] & \rightarrow & \mathbb{K}\left[x, x^{-1}\right] \\
z & \mapsto & x^{W_{\mathrm{o}}}
\end{array}
$$


is the prime (toric) ideal $P=\left(z^{V_{\mathrm{i}}^{+}}-z^{V_{\mathrm{i}}^{-}}\right):\left(z_{1} \ldots z_{n}\right)^{\infty}$ of dimension $r$ [25, Lemma 4.1, 4.2 and 12.2].

Assume $z \in\left(\mathbb{K}^{*}\right)^{n}$. For $\tilde{z}=\lambda^{A} \star z$ to be on the variety $\mathcal{P}$ of $P$ the components of $\tilde{z}^{V_{\mathrm{i}}}$ need to all be equal to 1 . Thus $\lambda^{A V_{\mathrm{i}}}=z^{-V_{\mathrm{i}}}$, that is, $\lambda^{H}=z^{-V_{\mathrm{i}}}$. Because of the triangular structure of $H$ we can always find $\lambda \in\left(\mathbb{K}^{*}\right)^{r}$ satisfying this equation. For any such $\lambda$ we then have $\tilde{z}=\left(\lambda^{A} \star z\right)^{V_{\mathfrak{i}} W_{\mathfrak{u}}+V_{\mathfrak{n}} W_{\mathfrak{o}}}$ since $V_{\mathfrak{i}} W_{\mathfrak{u}}+V_{\mathfrak{n}} W_{\mathfrak{d}}=I_{n}$ and so $\tilde{z}=\lambda^{H W_{\mathfrak{u}}} \star z^{V_{\mathfrak{i}} W_{\mathfrak{u}}+V_{\mathfrak{n}} W_{\mathfrak{o}}}=z^{-V_{\mathfrak{i}} W_{\mathfrak{u}}} \star z^{V_{\mathfrak{i}} W_{\mathfrak{u}}+V_{\mathfrak{n}} W_{\mathfrak{o}}}=z^{V_{\mathfrak{n}} W_{\mathfrak{o}}}$ by Proposition 3.1. Thus the intersection of the orbit of $z$ with the variety of $P$ exists, is unique and equal to $z^{V_{\mathfrak{n}} W_{\mathfrak{o}}}$.

From this description we deduce that the invariants $z^{V_{\mathrm{n}} W_{\mathrm{o}}}$ are actually the normalized invariants as defined in [10]. As such the rewriting of Theorem 4.2 applies to the more general class of smooth invariants. Furthermore, if the Hermite form of $A$ is $I_{r}$ there is a global moving frame for the group action, namely the equivariant map $\rho:\left(\mathbb{K}^{*}\right)^{n} \rightarrow\left(\mathbb{K}^{*}\right)^{r}$ given by $\rho(z)=z^{-V_{\mathfrak{i}}}$. The components $z^{V_{\mathfrak{n}} W_{\mathfrak{d}}}=\rho(z)^{A} \star z$ correspond then to the normalized invariants as originally defined in [6].

Example 4.7 Consider the scaling given by

$$
\left(z_{1}, z_{2}, z_{3}, z_{4}, z_{5}\right) \rightarrow\left(\frac{\eta}{\nu^{3}} z_{1}, \frac{\eta}{\mu} z_{2}, \eta z_{3}, \frac{\nu}{\eta \mu} z_{4}, \frac{\eta \nu}{\mu} z_{5}\right)
$$

an example used to illustrate dimensional analysis in [21]. In this case the matrix of exponents is

$$
A=\left[\begin{array}{rrrrr}
-3 & 1 & 1 & -1 & 1 \\
0 & -1 & 0 & -1 & -2 \\
1 & 0 & 0 & 1 & 1
\end{array}\right]
$$

The normal unimodular multiplier and its inverse are

$$
V=\left[\begin{array}{rrr|rr}
0 & 0 & 1 & -1 & -1 \\
0 & -1 & 0 & -1 & -2 \\
1 & 1 & 3 & -1 & -2 \\
0 & 0 & 0 & 1 & 0 \\
0 & 0 & 0 & 0 & 1
\end{array}\right] \text { and } W=\left[\begin{array}{rrrrr}
-3 & 1 & 1 & -1 & 1 \\
0 & -1 & 0 & -1 & -2 \\
1 & 0 & 0 & 1 & 1 \\
\hline 0 & 0 & 0 & 1 & 0 \\
0 & 0 & 0 & 0 & 1
\end{array}\right]
$$

Thus the rewrite rules are simply $z \rightarrow g^{W_{\mathfrak{o}}}=\left(1,1,1, g_{1}, g_{2}\right)$. By Theorem 4.6 the associated rational section is the variety $\left(z_{3}-1, z_{3}-z_{2}, z_{3}^{3}-z_{1}\right): z^{\infty}$. Combinations of the ideal generators show that this ideal is simply $\left(z_{1}-1, z_{2}-1, z_{3}-1\right)$. This favorable situation comes from the fact that the normal unimodular multiplier and its inverse have $a(n-r) \times r$ block of zeros at the bottom left.

The simplest case for the normalization of the unimodular multiplier $V$ occurs when the pivot rows of $V_{\mathfrak{n}}$ are the rows of an $(n-r)$-identity matrix. Assuming that the pivot rows appear at the end, a situation that can be arranged by permuting the columns of $A$ and therefore the order of the original variables, then the normal unimodular multiplier and its inverse are

$$
V=\left[\begin{array}{cc}
V_{\mathfrak{i}}^{*} & V_{\mathfrak{n}}^{*} \\
0 & I_{n-r}
\end{array}\right] \text { and } W=\left[\begin{array}{cc}
V_{\mathfrak{i}}^{*-1} & -V_{\mathfrak{i}}^{*-1} V_{\mathfrak{n}}^{*} \\
0 & I_{n-r}
\end{array}\right]
$$

The rewrite rules are then: $z \rightarrow g^{W_{\mathfrak{o}}}=\left(1, \ldots, 1, g_{1}, \ldots, g_{n-r}\right)$ which indicates that the equations for the section can be made simpler than in Theorem 4.6.

Proposition 4.8 If the normal unimodular multiplier $V$ of $A$ for its Hermite normal form is

$$
V=\left[\begin{array}{cc}
V_{\mathrm{i}}^{*} & V_{\mathfrak{n}}^{*} \\
0 & I_{n-r}
\end{array}\right]
$$

then the variety of $\left(z_{1}-1, \ldots, z_{r}-1\right)$ is a rational section to the scaling defined by $A$. There are then $n-r$ generating invariants $g_{r+1}^{*}, \ldots, g_{n}^{*}$ s.t. any other rational invariants can be written in terms of those with the substitution $\left(z_{1}, \ldots, z_{n}\right) \mapsto\left(1, \ldots, 1, g_{r+1}^{*}, \ldots, g_{n}^{*}\right)$. 
The proof proceeds by taking the power $\left(V_{\mathfrak{i}}^{*}\right)^{-1}$ of $\left(z^{V_{i}^{+}}-z^{V_{i}^{-}}\right)$. The components then belong to the ideal generated by the components of $\left(z^{V_{i}^{+}}-z^{V_{i}^{-}}\right)$and factor as a product of $\left(z_{1}-1, \ldots, z_{r}-1\right)$ with a monomial in $z$. Then $\left(1, \ldots, 1, g_{r+1}^{*}, \ldots, g_{n}^{*}\right)=z^{V_{\mathfrak{n}} \cdot W_{\mathfrak{o}}}=\left(1_{r}, z^{V_{\mathfrak{n}}}\right)=\left(1_{r}, g\right)$.

Note that the form (11) is the only possibility for the $n-r$ bottom rows of $V_{\mathfrak{i}}$ to be zero. As mentioned at the end of Subsection 2.2, the rows of $V_{\mathfrak{i}}$ being zero implies that the $n-r$ bottom rows of $V_{\mathfrak{n}}$ form a unimodular matrix, and hence can only be the identity.

\section{Determining scaling symmetries}

In the previous section we assumed that a scaling matrix is provided and we compute its rational invariants. In this section we consider the reverse problem. That is, we are given a finite set of rational functions and look for a maximal scaling matrix $A \in \mathbb{Z}^{r \times n}$ that leaves these functions invariant. This allows us to determine all the scaling symmetries of the dynamical systems studied in Sections 6 and 7.

Symmetries of differential systems are often determined through infinitesimal methods [21]. If we make the infinitesimal method specific to scaling symmetries, a solution can be achieved by computing the nullspace of a matrix [11]. However in that case we only have a local symmetry. In the case of a scaling symmetry of a dynamical system given by rational functions we can have a global picture.

Consider $f=\frac{p}{q} \in \mathbb{K}(z)$, where $p, q \in \mathbb{K}[z]$ are relatively prime, and pick $w$ in the support of $p$ or $q$. By Lemma 4.1, $A \cdot(v-w)=0$ for all $v$ in the support of $p$ and $q$. Let $K_{f}$ be the matrix whose columns consist of the vectors $v-w$ for all $v$ in the support of $p$ and $q$ (with $v \neq w$ ). Then $f=\frac{p}{q}$ is invariant for the scaling determined by $A$ if and only if $A \cdot K_{f}=0$. When $f$ is already a Laurent polynomial one should simply take $K_{f}$ to be the matrix of exponents of $f$ - thus considering $w=0$, the exponent of the denominator which is 1.

The scaling matrix $A$ is independent of the choice of $w$ in the support of $p$ or $q$. Indeed suppose $\alpha_{1}, \ldots, \alpha_{\ell}$ are the integer vectors of the form $v-w$ for all $v \neq w$ in the support of $p$ and $q$ and $\beta_{1}, \ldots, \beta_{\ell}$ are the integer vectors of the form $v-u$ with $v \neq u$ in the support and $w$ and $u$ distinct. Then there exists an index $k$ such that $\beta_{j}=\alpha_{j}-\alpha_{k}$ for all $j$. Then $A \cdot \alpha_{j}=0$ for all $j$ implies $A \cdot \beta_{j}=0$ for all $j$ (and conversely).

Consider a vector of rational functions $F(z)=\left[F_{1}(z), \ldots, F_{m}(z)\right]$. To each component $F_{i}$ we can associate a matrix $K_{i}$ as previously described. Let $K=\left[K_{1}, \ldots, K_{m}\right]$. Then the necessary and sufficient condition for $F$ to be an invariant map for the scaling defined by $A$ is that $A \cdot K=0$. For this we have the following, which is a simple variation of a proposition found in [5, pg 72].

Proposition 5.1 Suppose $K \in \mathbb{Z}^{n \times m}$ is a matrix of integers and that $U \in \mathbb{Z}^{n \times n}$ is a unimodular matrix such that $U \cdot K$ is in row Hermite normal form

$$
U \cdot K=\left[\begin{array}{c}
K_{1} \\
0
\end{array}\right]
$$

having exactly $r$ zero rows. Let $A$ be the last $r$ rows of $U$. Then

(i) The column Hermite normal form of $A$ is $\left[I_{r}, 0\right]$.

(ii) An integer matrix $B$ satisfies $B \cdot K=0$ iff there exists an integer matrix $M$ such that $B=M \cdot A$.

Proof: Let $V$ be the inverse of $U$. Then $A \cdot V=\left[0, I_{r}\right]$ and so permuting the columns of $V$ gives a unimodular multiplier having trivial Hermite normal form. This gives (i). Property (ii) follows from the fact that $A$ is a basis for the integer lattice given by left kernel of $K$.

The first property implies in particular that $A$ is of full row rank. It furthermore defines a scaling without isotropy (cf. Proposition 3.3). 
The second property shows the maximality of the scaling found. If $A^{*}$ is another matrix with the same property then there is a unimodular matrix $U^{*}$ such that $A^{*}=U^{*} \cdot A$. Otherwise $B=U^{*} \cdot A$ has either lower rank or has a nontrivial Hermite normal form.

Example 5.2 In order to find the scaling symmetry of the prey-predator model presented in the introduction we need to determine the scalings that leave invariant the two rational functions which are the components of

$$
F=\left[t\left(r\left(1-\frac{n}{K}\right)-k \frac{p}{n+d}\right), s t\left(1-h \frac{p}{n}\right)\right] .
$$

The first step is to normalize one of the terms of the denominators. For instance we consider:

$$
F=\left[\frac{r t+d t n^{-1}-r K^{-1} t n-r K^{-1} d t-k t n^{-1} p}{1+d n^{-1}}, \frac{s t-h n^{-1} s t p}{1}\right] .
$$

We can then form the matrix $K$ with the non trivial exponents. With variable order $(r, K, k, s, h, d, s, t, n, p)$ this matrix is:

$$
K=\left[\begin{array}{rrrrrrrr}
0 & 1 & 1 & 1 & 1 & 0 & 0 & 0 \\
0 & 0 & 0 & 0 & 0 & 0 & 0 & 1 \\
0 & 0 & 0 & -1 & -1 & 0 & 0 & 0 \\
0 & 0 & 0 & 0 & 0 & 0 & 1 & 1 \\
0 & 0 & 0 & 0 & 0 & 1 & 0 & 0 \\
1 & 0 & 1 & 0 & 1 & 0 & 0 & 0 \\
0 & 1 & 1 & 1 & 1 & 1 & 1 & 1 \\
-1 & 0 & -1 & 1 & 0 & -1 & 0 & -1 \\
0 & 0 & 0 & 0 & 0 & 1 & 0 & 1
\end{array}\right]
$$

Here the first 6 columns of $K$ are determined from the exponents of the first component of $F$ and the last 2 columns are determined from the second component.

Applying Proposition 5.1 we determine a $9 \times 9$ unimodular matrix $U$ such that $U \cdot K$ is in row Hermite normal form. The row Hermite normal form here has 3 zero rows at the end. We thus retain from $U$ the bottom three rows. The scalings leaving $F$ invariant is thus given by the matrix

$$
A=\left[\begin{array}{rrrrrrrrr}
-1 & 0 & 0 & -1 & -1 & 0 & 1 & 0 & 0 \\
0 & 1 & 1 & 0 & 1 & 1 & 0 & 1 & 0 \\
0 & -1 & 0 & 0 & -1 & 0 & 0 & 0 & 1
\end{array}\right]
$$

\section{Dynamical Systems}

In this section we consider dynamical systems of the form

$$
\frac{d z}{d t}=G(t, z)
$$

where $z=\left(z_{1}, \ldots, z_{n}\right)$ is a vector of variables dependent on $t$ and $G$ is a rational map $\mathbb{R} \times \mathbb{R}^{n} \rightarrow \mathbb{R}^{n}$. We examine the simplification that can be obtained when such a system has a scaling symmetry.

The symmetry of a differential system is a group of transformations that leaves the solution set invariant [21]. We first study the case where the scaling acts only on the $z$ variables. We then consider the more general case where the scaling acts on $t$ as well as on $z$.

There is a common understanding that a system with symmetry can be reduced. The reduced system is the dynamical system induced on the invariants of the group action describing the symmetry. The more difficult part is to recover the solutions of the original system from the solutions of the reduced system. 
In the case studied here, we show how to obtain directly a reduced system and how the solution of the original system can be deduced from the solution of the reduced system with some additional quadratures. The reduced system and the quadratures are very simply constructed from a unimodular multiplier, and its inverse, for the Hermite normal form of the scaling matrix.

The methodological approach of symmetry reduction in [1] applies to exterior differential systems. It is specialised for symmetry reduction of ordinary differential equations in [7]. It requires first a quotient map and a cross-section. In the case of scaling symmetries those are provided by the monomial maps defined by $V_{\mathfrak{i}}$ and $W_{\mathfrak{d}}$ respectively. One then needs to find the semi-basic forms and the ODE structure of the reduced system. The solution of the original system are then recovered by determining and solving equations of Lie type. For scalings, the underlying group is Abelian so those equations can be brought to independent quadratures. The constructive content of those steps does not appear as straightforward.

The method in [18, Section 6] applies the ingredients of the moving frame as initially introduced in [6]. It requires a (local) cross-section as input and a companion equation. In the case of scalings, there is self evident companion equation that we actually use here. The differential equations are then rewritten in terms of the normalized invariants and one needs to spot a differentially generating subset among those, with the possibility of requiring syzygies. The solution of the original equations are then obtained thanks to the solutions of the differential system bearing on the moving frame. This latter is of Lie type. It is the set of quadratures we obtain, when there is no isotropy.

\subsection{Symmetry on the dependent variables}

Consider a scaling on $\mathbb{K}^{n}$ defined by $A \in \mathbb{Z}^{r \times n}$. The condition for the scaling defined by $A$ to be a symmetry of the differential system (13) is that $G$ be equivariant with respect to $z$, that is, $G\left(t, \lambda^{A} \star x\right)=\lambda^{A} \star G(t, z)$. In this case, if $z(t)$ is a solution of (13) then, for any $\lambda \in\left(\mathbb{R}^{*}\right)^{r}, \lambda^{A} \star z(t)$ is also a solution.

For the rest of this subsection we simply write $G(z)$, omitting $t$, even though we do not assume that $G$ is independent of $t$. Rather the notation is used since only the scaling is assumed to act trivially on $t$. With the notation

$$
z^{-1}=\left[z_{1}^{-1}, \ldots, z_{n}^{-1}\right]
$$

we have that $z^{-1} \star G(z)$ is an invariant map so that there is no loss of generality in considering the dynamical system

$$
\frac{d z}{d t}=z \star F(z) \quad \text { where } F\left(\lambda^{A} \star z\right)=F(z)
$$

Let $V=\left[V_{\mathfrak{i}}, V_{\mathfrak{n}}\right]$ be a unimodular matrix with inverse $W=\left[\begin{array}{l}W_{\mathfrak{u}} \\ W_{\mathfrak{d}}\end{array}\right]$ such that $A \cdot V$ is in Hermite normal form. We consider the new variables $x=z^{V_{\mathrm{i}}}$ and $y=z^{V_{\mathfrak{n}}}$. The variables $y$ stand for the invariants of the scaling, while the auxiliary variables $x$ stand for a moving frame up to isotropy (Theorems 4.2 and 4.6). The dynamics for $x$ and $y$ are obtained by application of the following useful lemma.

Lemma 6.1 Suppose $z=\left[z_{1}, \ldots, z_{n}\right]$ is a vector of functions of time $t$ and $B \in \mathbb{Z}^{n \times k}$ is a matrix of integers. Then

$$
\frac{d}{d t}\left(z^{B}\right)=z^{B} \star\left[\left(z^{-1} \star \frac{d z}{d t}\right) \cdot B\right] .
$$

PROOF: Suppose first that $b=\left[b_{1}, \ldots, b_{n}\right]^{T}$ is an arbitrary column vector of integers. Then $z^{b}=z_{1}^{b_{1}} \cdots z_{n}^{b_{n}}$ 
and so

$$
\begin{aligned}
\frac{d}{d t}\left(z^{b}\right) & =b_{1} \frac{z^{b}}{z_{1}} \cdot \frac{d z_{1}}{d t}+\cdots+b_{n} \frac{z^{b}}{z_{n}} \cdot \frac{d z_{1}}{d t} \\
& =z^{b}\left(\frac{b_{1}}{z_{1}} \cdot \frac{d z_{1}}{d t}+\cdots+\frac{b_{n}}{z_{n}} \cdot \frac{d z_{1}}{d t}\right) \\
& =z^{b}\left[\left(z^{-1} \star \frac{d z}{d t}\right) \cdot b\right] .
\end{aligned}
$$

The result then follows by applying (16) to each column of $B$.

Proposition 6.2 Consider a map $F: \mathbb{R}^{n} \rightarrow \mathbb{R}^{n}$ that is invariant under the scaling defined by $A \in \mathbb{Z}^{r \times n}$. Let $V=\left[V_{\mathfrak{i}}, V_{\mathfrak{n}}\right]$ be a unimodular matrix with inverse $W=\left[\begin{array}{l}W_{\mathfrak{u}} \\ W_{\mathfrak{o}}\end{array}\right]$ such that $A \cdot V$ is in Hermite normal form. If $z(t)$ is a solution of $\frac{d z}{d t}=z \star F(z)$ where none of the components vanish then

$$
[x(t), y(t)]=\left[z(t)^{V_{\mathrm{i}}}, z(t)^{V_{\mathrm{n}}}\right]
$$

is a solution of the dynamical system:

$$
\begin{aligned}
& \frac{d y}{d t}=y \star F\left(y^{W_{\mathfrak{}}}\right) \cdot V_{\mathfrak{n}} \\
& \frac{d x}{d t}=x \star F\left(y^{W_{\mathfrak{}}}\right) \cdot V_{\mathfrak{i}} .
\end{aligned}
$$

PRoOF: From Lemma 6.1 we have that $\frac{d y}{d t}=y \star\left(F(z) \cdot V_{\mathfrak{n}}\right)$ and, since $F$ is invariant $F(z)=F\left(z^{V_{\mathfrak{n}} \cdot W_{\mathfrak{}}}\right)=$ $F\left(y^{W_{0}}\right)$ by Theorem 4.2. The same argument gives equation (18).

System (17) is the reduced system: it is the dynamical system bearing on the $n-r$ variables which are intrinsically generating invariants of the scaling. System (18) is an auxiliary system providing the dynamic system of the moving frame as defined in [6], up to isotropy. It is actually a quadrature. For a given solution to the system (17), a solution to (18) is obtained by integration:

$$
x=\exp \left(\int F\left(y^{W_{\mathrm{o}}}\right) \cdot V_{\mathrm{i}} d t\right) .
$$

The coupled system (17-18) thus lends itself better to solving or to analysis. The next result shows how we can recover the solutions of the original system from the solutions of the reduced system with the help of the auxiliary system.

Theorem 6.3 Consider a map $F: \mathbb{R}^{n} \rightarrow \mathbb{R}^{n}$ that is invariant under the scaling defined by $A \in \mathbb{Z}^{r \times n}$. Let $V=\left[V_{\mathfrak{i}}, V_{\mathfrak{n}}\right]$ be a unimodular matrix with inverse $W=\left[\begin{array}{l}W_{\mathfrak{u}} \\ W_{\mathfrak{d}}\end{array}\right]$ such that $A \cdot V$ is in Hermite normal form. If $y(t)$ and $x(t)$ are solutions of the dynamical systems

$$
\frac{d y}{d t}=y \star F\left(y^{W_{\mathfrak{o}}}\right) \cdot V_{\mathfrak{n}}, \quad \frac{d x}{d t}=x \star F\left(y^{W_{\mathfrak{o}}}\right) \cdot V_{\mathfrak{i}},
$$

where none of the components vanish, then $z(t)=[x(t), y(t)]^{W}=x(t)^{W_{\mathfrak{u}}} \star y(t)^{W_{\mathrm{\diamond}}}$ is a solution of the dynamical system

$$
\frac{d z}{d t}=z \star F(z) .
$$


PROOF: By Lemma 6.1 we have

$$
\frac{d}{d t}(z(t))=\frac{d}{d t}\left([x(t), y(t)]^{W}\right)=z(t) \star\left([x(t), y(t)]^{-1} \star \frac{d}{d t}([x(t), y(t)]) \cdot W\right) .
$$

From $\frac{d}{d t}([x(t), y(t)])=[x(t), y(t)] \star F\left(y(t)^{W_{\mathfrak{\jmath}}}\right) \cdot V$ and $V \cdot W=I_{n}$ we obtain $\frac{d}{d t}(z(t))=z(t) \star F\left(y(t)^{W_{\mathfrak{o}}}\right)$. Since $W \cdot V_{\mathfrak{n}}=\left[\begin{array}{c}0 \\ I_{n-r}\end{array}\right]$ we have $z(t)^{V_{\mathfrak{n}}}=[x(t), y(t)]^{W \cdot V_{\mathfrak{n}}}=y(t)$, and so, by Theorem $4.2, F\left(y(t)^{W_{\mathfrak{o}}}\right)=F(z(t))$ since $F$ is an invariant.

Example 6.4 Consider the dynamical system

$$
\frac{d z_{1}}{d t}=z_{1}\left(1+z_{1} z_{2}\right), \quad \frac{d z_{2}}{d t}=z_{2}\left(\frac{1}{t}-z_{1} z_{2}\right)
$$

Then $A, V$ and $W$ defined by

$$
A=\left[\begin{array}{ll}
1 & -1
\end{array}\right], \quad V=\left[\begin{array}{ll}
1 & 1 \\
0 & 1
\end{array}\right], \quad W=\left[\begin{array}{cc}
1 & -1 \\
0 & 1
\end{array}\right] .
$$

defines a scaling symmetry for this system with $V$ a unimodular matrix such that $A \cdot V$ is in Hermite normal form and $W$ is the inverse of $V$.

We introduce, as new variables, the invariant of the scaling $y=z^{V_{\mathfrak{n}}}=z_{1} z_{2}$ and the auxiliary variable $x=z^{V_{\mathrm{i}}}=z_{1}$. The induced dynamical system for those variables are, on one hand, the reduced system consisting of a single equation - and a quadrature:

$$
\frac{d y}{d t}=y\left(1+\frac{1}{t}\right), \quad \frac{1}{x} \frac{d x}{d t}=1+y .
$$

It is reasonably easy to write the solution of this linear differential system:

$$
y(t)=c_{1} t e^{t}, \quad x(t)=c_{2} \exp \left(t+c_{1}(t-1) e^{2 t}\right) .
$$

We can thus provide the solutions of the original system as $\left[z_{1}(t), z_{2}(t)\right]=[x(t), y(t)]^{W}$, which is

$$
z_{1}(t)=x(t)=c_{2} \exp \left(t+c_{1}(t-1) e^{t}\right), \quad z_{2}(t)=\frac{y(t)}{x(t)}=\frac{c_{1}}{c_{2}} t \exp \left(c_{1}(t-1) e^{t}\right) .
$$

\subsection{General case}

Consider a scaling on $\mathbb{K}^{n+1}$ defined by $\bar{A} \in \mathbb{Z}^{r \times(n+1)}$. The condition for the scaling defined by $\bar{A}$ to be a symmetry of the differential system $(13)$ is that $F(t, z)=t z^{-1} \star G(t, z)$ be an invariant map for the scaling. Without loss of generality we therefore write our dynamical system in the form

$$
t \frac{d z}{d t}=z \star F(t, z)
$$

where $F: \mathbb{R} \times \mathbb{R}^{n} \rightarrow \mathbb{R}^{n}$ is an invariant map for the scaling defined by $A \in \mathbb{Z}^{r \times(n+1)}$.

We write $\bar{A}=\left[A_{0}, A\right]=\left[A_{0}, A_{1}, \ldots, A_{n}\right]$ so that the scaling is given by

$$
\lambda^{\bar{A}} \star[t, z]=\left[\lambda^{A_{0}} t, \lambda^{A} z\right]=\left[\lambda^{A_{0}} t, \lambda^{A_{1}} z_{1}, \ldots, \lambda^{A_{n}} z_{n}\right] .
$$


It is actually convenient to introduce an additional dependent variable $z_{0}$, add an equation for it, and keep time invariant in a first step. We set $\bar{F}=[1, F]$ and $\bar{z}=\left(z_{0}, z_{1}, \ldots, z_{n}\right)$ and consider the dynamical system:

$$
t \frac{d \bar{z}}{d t}=\bar{z} \star \bar{F}(\bar{z})
$$

The first equation of this dynamical system is simply $t \frac{d z_{0}}{d t}=z_{0}$ so that $t^{-1} z_{0}$ is constant. If $\bar{z}(t)=$ $\left[\bar{z}_{0}(t), \bar{z}_{1}(t), \ldots, \bar{z}_{n}(t)\right]$ is a solution of $(21)$ and the constant $c=t^{-1} \bar{z}_{0}(t)$ is not zero, then $z(t)=\left[\bar{z}_{1}\left(\frac{t}{c}\right), \ldots, \bar{z}_{n}\left(\frac{t}{c}\right)\right]$ is a solution of $(20)$. Conversely, if $z(t)=\left[z_{1}(t), \ldots, z_{n}(t)\right]$ is a solution of $(20)$ then $\left[t, z_{1}(t), \ldots, z_{n}(t)\right]$ is a solution of $(21)$.

If we set $s=\ln (t)$ then system $(21)$ can be rewritten as $\frac{d \bar{z}}{d s}=\bar{z} \star \bar{F}(\bar{z})$. We can then apply the reduction of Theorem 6.3. We can also keep $t$ as the independent variable. The statement and the proof are completely analogous.

Theorem 6.5 Consider a map $F: \mathbb{R} \times \mathbb{R}^{n} \rightarrow \mathbb{R}^{n}$ that is invariant under the scaling defined by $\bar{A} \in \mathbb{Z}^{r \times(n+1)}$. Let $V=\left[V_{\mathfrak{i}}, V_{\mathfrak{n}}\right]$ be a unimodular matrix with inverse $W=\left[\begin{array}{l}W_{\mathfrak{u}} \\ W_{\mathfrak{d}}\end{array}\right]$ such that $\bar{A} \cdot V$ is in Hermite normal form. Assume $y(t)$ and $x(t)$ are solutions of the dynamical systems

$$
\begin{aligned}
t \frac{d y}{d t} & =y \star \bar{F}\left(y^{W_{\mathfrak{o}}}\right) \cdot V_{\mathfrak{n}}, \\
t \frac{d x}{d t} & =x \star \bar{F}\left(y^{W_{\mathfrak{o}}}\right) \cdot V_{\mathfrak{i}},
\end{aligned}
$$

where $\bar{F}=[1, F]$, having the property that none of their components vanish. If $\left[\tilde{z}_{0}(t), \tilde{z}_{1}(t) \ldots, \tilde{z}_{n}(t)\right]=$ $[x(t), y(t)]^{W}$ then $t^{-1} \tilde{z}_{0}(t)$ is a nonzero constant $c$ and $z(t)=\left[\tilde{z}_{1}\left(\frac{t}{c}\right) \ldots, \tilde{z}_{n}\left(\frac{t}{c}\right)\right]$ is a solution of the dynamical system

$$
t \frac{d z}{d t}=z \star F(z)
$$

The system (22) is the reduced system having $n+1-r$ variables, which correspond to the generating set of invariants and can be read from $V$. The change of time $s=\ln (t)$ makes it an autonomous system. In addition, (23) is a quadrature. If $y(t)$ is a solution to (22) then the complete solution is obtained by integration:

$$
x=\exp \left(\int F\left(y^{W_{\mathfrak{o}}}\right) \cdot V_{\mathfrak{i}} t^{-1} d t\right) .
$$

Example 6.6 Consider the dynamical system given by

$$
t \frac{d z_{1}}{d t}=z_{1}\left(-\frac{2}{3}+\frac{1}{3} z_{1}^{5} z_{2}\right) \quad t \frac{d z_{2}}{d t}=z_{2}\left(\frac{10}{3}-\frac{2}{3} z_{1}^{5} z_{2}+\frac{z_{1}^{2} z_{2}}{t}\right) .
$$

This dynamical system is invariant under the scaling defined by the matrix $A=\left[\begin{array}{lllll}3 & -1 & 5\end{array}\right]$. The normal unimodular multiplier and its inverse for the Hermite normal form of $A$ are given by

$$
V=\left[\begin{array}{rrr}
1 & 1 & -1 \\
2 & 3 & 2 \\
0 & 0 & 1
\end{array}\right], \quad W=\left[\begin{array}{rrr}
3 & -1 & 5 \\
-2 & 1 & -4 \\
0 & 0 & 1
\end{array}\right]
$$

We see that $\bar{F}\left(t, z_{1}, z_{2}\right)=\left[1,-\frac{2}{3}+\frac{z_{1}^{5} z_{2}}{3}, \frac{10}{3}-\frac{2}{3} z_{1}^{5} z_{2}+\frac{z_{1}^{2} z_{2}}{t}\right]$ and $y^{W_{\mathfrak{o}}}=\left(y_{1}, y_{2}\right)^{W_{\mathfrak{o}}}=\left(\frac{1}{y_{1}^{2}}, y_{1}, \frac{y_{2}}{y_{1}^{4}}\right)$ and so

$$
\bar{F}\left(y^{W_{\mathfrak{o}}}\right)=\left[1,-\frac{2}{3}+\frac{y_{1} y_{2}}{3}, \frac{10}{3}-\frac{2}{3} y_{1} y_{2}+y_{2}\right] \text {. }
$$


The reduced dynamical system is thus given by

$$
t \frac{d y_{1}}{d t}=y_{1}\left(y_{1} y_{2}-1\right) \quad \text { and } \quad t \frac{d y_{2}}{d t}=y_{2}\left(1+y_{2}\right)
$$

and the auxiliary equation is

$$
t \frac{d x}{d t}=x\left(\frac{2}{3} y_{1} y_{2}-\frac{1}{3}\right) .
$$

Here $y_{1}$ and $y_{2}$ represent the invariants $t z_{1}{ }^{3}$ and $\frac{z_{1}^{2} z_{2}}{t}$, respectively, while the auxiliary variable $x$ represents $t z_{1}^{2}$.

Using Maple one obtains closed form solutions for these equations, solving first for $y_{2}$, then $y_{1}$ and $x$ :

$$
\begin{aligned}
y_{2}(t) & =\frac{t}{c_{1}-t} \\
y_{1}(t) & =\frac{c_{1}}{t\left(\ln \left(t-c_{1}\right)-\ln (t)+c_{2}\right)} \\
x(t) & =\frac{c_{3}}{t^{1 / 3}\left(\ln \left(t-c_{1}\right)-\ln (t)+c_{2}\right)^{2 / 3}}
\end{aligned}
$$

with $c_{1}, c_{2}, c_{3}$ arbitrary constants. A solution to the original dynamical system is therefore given by

$$
\begin{aligned}
\left(z_{0}(t), z_{1}(t), z_{2}(t)\right) & =(x(t), y(t))^{W}=\left(\frac{x(t)^{3}}{y_{1}(t)^{2}}, \frac{y_{1}(t)}{x(t)}, \frac{x(t)^{5} y_{2}(t)}{y_{1}(t)^{4}}\right) \\
& =\left(\frac{c_{3}^{3}}{c_{1}^{2}} t, \frac{c_{1}}{c_{3} t^{2 / 3}\left(\ln \left(t-c_{1}\right)-\ln (t)+c_{2}\right)^{1 / 3}}, \frac{c_{3}^{5} t^{10 / 3}\left(\ln \left(t-c_{1}\right)-\ln (t)+c_{2}\right)^{2 / 3}}{c_{1}^{4}\left(-t+c_{1}\right)}\right) .
\end{aligned}
$$

Substituting $t \rightarrow t / c$ with $c=\frac{c_{3}^{3}}{c_{1}^{2}}$, simplifying and renaming the constants gives the solution of the original system as

$$
z_{1}(t)=\frac{a^{1 / 3}}{t^{2 / 3}(\ln (t-a)-\ln (t)+b)^{1 / 3}}, \quad z_{2}(t)=\frac{t^{10 / 3}(\ln (t-a)-\ln (t)+b)^{2 / 3}}{a^{2 / 3}(a-t)}
$$

with $a, b$ arbitrary constants. We note that this solution is considerably simpler than that produced by Maple.

\section{Models with parameters}

Dynamical systems are a standard tool in modeling. The model bears on some state variables $z_{1}, \ldots, z_{q}$ that evolve with time $t$ and the equations are written with some constant parameters $c_{1}, \ldots, c_{p}$ that describe the media. The parameterized dynamical system can be written

$$
\frac{d z}{d t}=G(c, t, z)
$$

Biological models typically come with more parameters than are relevant for a qualitative analysis: there is often a way to group parameters without qualitative change to the solution [20]. The rule of thumb used then can often be explained by a scaling symmetry. This was the case of the predator-prey model in the introduction.

In this section we apply the results of the previous section to reduce the number of parameters in the presence of a scaling symmetry. With a series of classical models we demonstrate our algorithmic approach to the reduction of parameters: first compute the scaling symmetry, then produce the reduced system and the correspondence with the solution of the original system. Each example illustrate a different aspect of the reduction proposed. 


\subsection{Symmetry reduction of the number of parameters}

Note that (24) can be recast into (13) by extending the system with the equations $\frac{d c}{d t}=0$. The matrix $A \in \mathbb{Z}^{r \times n}, n=p+q+1$, defines a scaling symmetry of (24) if the map $F(c, t, z)=t z^{-1} \star G(c, t, z)$ is an invariant: $F\left(\lambda^{A} \star(c, t, z)\right)=F(c, t, z)$.

We assume that the normal unimodular multiplier for the Hermite normal form of $A$ has the form ${ }^{4}$ :

$$
V=\left[\begin{array}{c|ccc}
V_{\mathfrak{i}}^{*} & V_{\mathfrak{n}}^{\dagger} & V_{\mathfrak{n}}^{0} & V_{\mathfrak{n}}^{\ddagger} \\
\hline 0_{1 \times r} & 0_{1 \times(p-r)} & 1 & 0_{1 \times q} \\
0_{q \times r} & 0_{q \times(p-r)} & 0_{q \times 1} & I_{q}
\end{array}\right], \text { and hence with inverse } W=\left[\begin{array}{ccc} 
& W_{\mathfrak{u}} & \\
3 & & \\
W_{\mathfrak{d}}^{\dagger} & W_{\mathfrak{d}}^{0} & W_{\mathfrak{d}}^{\ddagger} \\
0_{1 \times p} & 1 & 0_{1 \times q} \\
0_{q \times p} & 0_{q \times 1} & I_{q}
\end{array}\right],
$$

where $V_{\mathfrak{i}}^{*}, V_{\mathfrak{n}}^{\dagger}, V_{\mathfrak{n}}^{0}$ and $V_{\mathfrak{n}}^{\ddagger}$ have $p$ rows while $W_{\mathfrak{d}}^{\dagger}, W_{\mathfrak{d}}^{0}, W_{\mathfrak{d}}^{\ddagger}$ have $p-r$ rows. As we shall show, we can then reduce the number of parameters by $r$. The appropriate combinations of parameters to consider are read from $V$ and the reduced system is obtained by a simple substitution given by $W$.

We could write an explicit scheme for reducing by $r$ the number of parameters if the unimodular multiplier simply had a $(q+1) \times p$ block of zeros in the bottom left corner, that is, was of the form:

$$
\left[\begin{array}{cc|c}
V_{\mathfrak{i}}^{*} & V_{\mathfrak{n}}^{\dagger} & V_{\mathfrak{n}}^{\ddagger} \\
\hline 0_{(q+1) \times p} & & V_{\mathfrak{n}}^{\sharp}
\end{array}\right]
$$

However, in this case $V_{\mathfrak{n}}^{\sharp}$ would be unimodular and the normal unimodular multiplier would be of the form (25). The class of systems that can be reduced is thus not larger.

In light of Theorem 6.5 we introduce

- $\mathfrak{c}=\left(\mathfrak{c}_{1}, \ldots, \mathfrak{c}_{p-r}\right)=\left(c_{1}, \ldots, c_{p}\right)^{V_{\mathfrak{n}}^{\dagger}}$

- $\mathfrak{t}=\left(c_{1}, \ldots, c_{p}\right)^{V_{\mathfrak{n}}^{0}} t$

- $\mathfrak{z}=\left(\mathfrak{z}_{1}, \ldots, \mathfrak{z}_{q}\right)=\left(c_{1}, \ldots, c_{p}\right)^{V_{\mathfrak{n}}^{\ddagger}} \star\left(z_{1}, \ldots, z_{q}\right)$

The reduced system has $p-r$ parameters, $\mathfrak{c}$, and $q$ state variables, $\mathfrak{z}$. It is explicitly given by:

$$
\frac{d \mathfrak{z}}{d \mathfrak{t}}=\mathfrak{c}^{W_{\mathfrak{o}}^{0}} \mathfrak{c}^{-W_{\mathfrak{o}}^{\ddagger}} \star G\left(\mathfrak{c}^{W_{\mathfrak{o}}^{\dagger}}, \mathfrak{c}^{W_{\mathfrak{o}}^{0}} \mathfrak{t}, \mathfrak{c}^{W_{\mathfrak{o}}^{\ddagger}} \star \mathfrak{z}\right) .
$$

In other words, the reduced system is obtained with the following substitution:

$$
c \mapsto \mathfrak{c}^{W_{\mathfrak{o}}^{\dagger}}, t \mapsto \mathfrak{c}^{W_{\mathfrak{o}}^{0}} \mathfrak{t}, z \mapsto \mathfrak{c}^{W_{\mathfrak{o}}^{\ddagger}} \star \mathfrak{z}
$$

If $(c, z(t))$ is a solution of (24) without vanishing components then $\left(c^{V_{n}^{\dagger}}, c^{V_{n}^{\ddagger}} z\left(c^{-V_{n}^{0}} \mathfrak{t}\right)\right)$ is a solution of (26). Conversely if $(\mathfrak{c}, \mathfrak{z}(\mathfrak{t}))$ is a solution of $(26)$ with no zero components then $\left(\mathfrak{d}^{W_{\mathfrak{u}}} \star\left(\mathfrak{c}^{W_{\mathfrak{o}}^{\dagger}}, \mathfrak{c}^{W_{\mathfrak{o}}^{\ddagger}} \star \mathfrak{z}\left(c^{-W_{\mathfrak{o}}^{0}} t\right)\right)\right)$ is a solution of $(24)$, for any constants $\mathfrak{d}=\left(\mathfrak{d}_{1}, \ldots, \mathfrak{d}_{r}\right)$ without vanishing components.

\footnotetext{
${ }^{4}$ This was the case in the many $(>40)$ models from mathematical biology we examined.
} 


\subsection{Verhulst model of logistic growth}

Consider first the Verhulst model of logistic growth [20, Section 1.1]

$$
\frac{d n}{d t}=r n\left(1-\frac{n}{k}\right)
$$

The scaling symmetries of this system are the scalings that leave the Laurent polynomial $r^{1} k^{0} t^{1} n^{0}-r^{1} k^{-1} t^{1} n^{1}$ invariant. Following Section 5 we form the matrix $K$ of its exponents:

$$
K=\left[\begin{array}{rr}
1 & 1 \\
0 & -1 \\
1 & 1 \\
0 & 1
\end{array}\right]
$$

Applying Proposition 5.1 one determines the matrix $A$ that describes the scaling symmetry for this system,

$$
A=\left[\begin{array}{rrrr}
-1 & 0 & 1 & 0 \\
0 & 1 & 0 & 1
\end{array}\right]
$$

One can indeed see that the system is invariant if we make any of the following change of variables parameterized by $(\mu, \nu)$

$$
r \mapsto \mu^{-1} r, k \mapsto \nu k, t \mapsto \mu t, n \mapsto \nu n
$$

The normal unimodular multiplier, and its inverse, for the Hermite normal form of $A$ are:

$$
V=\left[\begin{array}{rr|rr}
-1 & 0 & 1 & 0 \\
0 & 1 & 0 & -1 \\
0 & 0 & 1 & 0 \\
0 & 0 & 0 & 1
\end{array}\right], \quad \text { and } \quad W=\left[\begin{array}{rrrr}
-1 & 0 & 1 & 0 \\
0 & 1 & 0 & 1 \\
\hline 0 & 0 & 1 & 0 \\
0 & 0 & 0 & 1
\end{array}\right]
$$

With the substitution read from $W_{\mathfrak{d}}, r \mapsto 1, k \mapsto 1, t \mapsto \mathfrak{t}, n \mapsto \mathfrak{n}$, we obtain the reduced system:

$$
\frac{d \mathfrak{n}}{d t}=\mathfrak{n}(1-\mathfrak{n})
$$

We can combine the general solution $\mathfrak{n}(\mathfrak{t})=\left(1+c e^{-\mathfrak{t}}\right)^{-1}$ of the reduced system and two constants $(\mathfrak{r}, \mathfrak{k})$ into the general solution of the original system as follows from $W$ :

$$
r=\frac{1}{\mathfrak{r}}, k=\mathfrak{k}, n(t)=\mathfrak{k} \mathfrak{n}\left(\frac{t}{\mathfrak{r}}\right)=\mathfrak{k}\left(1+c e^{-\frac{t}{\mathfrak{r}}}\right)^{-1} .
$$

Conversely any solution of the original system $n(t)=k\left(1+c e^{-r t}\right)^{-1}$ provides a solution of the reduced system by taking $\mathfrak{n}(\mathfrak{t})=\frac{1}{k} n\left(\frac{\mathfrak{t}}{r}\right)$ as dictated by $V$.

\subsection{Lotka-Volterra equations}

The scaling symmetry reduction of a given dynamical system is not unique. For example, the dynamical system governing a reaction kinetics described in [20, Section 6.6]

$$
\frac{d x}{d t}=k_{1} a x-k_{2} x y, \quad \frac{d y}{d t}=k_{2} x y-k_{3} y
$$

has parameters $\left(a, k_{1}, k_{2}, k_{3}\right)$ and variables $(x, y)$. In $[20$, Section 6.6] it is reduced to the Lotka-Volterra equations

$$
\frac{d u}{d \tau}=u(1-v), \quad \frac{d v}{d \tau}=\alpha v(u-1)
$$


using the variables

$$
\alpha=\frac{k_{3}}{k_{1} a}, \quad \tau=a k_{1} t, \quad u=\frac{k_{2}}{k_{3}} x, \quad v=\frac{k_{2}}{k_{1} a} y .
$$

This change of variables can be obtained through the general scheme we described in Section 6 . When we see it this way we have a simple way of rewriting the system in terms of these new variables. It is given by an explicit substitution:

$$
a \mapsto 1, k_{1} \mapsto 1, k_{2} \mapsto 1, k_{3} \mapsto \alpha, t \mapsto \tau, x \mapsto \alpha u, y \mapsto v
$$

The first step of the algorithm is to determine the scaling symmetry of the dynamical system: these are the scalings that leave the components of $\left[t\left(k_{1} a-k_{2} y\right), t\left(k_{2} x-k_{3}\right)\right]$ invariant. To determine those we form the matrix $K$ of the exponents:

$$
K=\left[\begin{array}{llll}
1 & 0 & 0 & 0 \\
1 & 0 & 0 & 0 \\
0 & 1 & 1 & 0 \\
0 & 0 & 0 & 1 \\
1 & 1 & 1 & 1 \\
0 & 0 & 1 & 0 \\
0 & 1 & 0 & 0
\end{array}\right]
$$

A basis of the lattice left kernel of $K$ provides the maximal scaling symmetry of the system (Section 5 ). It is given by the matrix:

$$
A=\left[\begin{array}{rrrrrrr}
0 & -1 & -1 & -1 & 1 & 0 & 0 \\
-1 & 1 & 0 & 0 & 0 & 0 & 0 \\
2 & -2 & -1 & 0 & 0 & 1 & 1
\end{array}\right]
$$

By Theorem 4.2 a minimal set of generating invariants for this scaling is given by any unimodular multiplier $V$ such that $A \cdot V$ is in Hermite normal form ${ }^{5}$. The unimodular multiplier underlying the above choice of new variables given above is:

$$
V=\left[\begin{array}{rrr|rrrr}
-1 & 1 & 1 & -1 & 1 & 0 & -1 \\
-1 & 2 & 1 & -1 & 1 & 0 & -1 \\
0 & -2 & -1 & 0 & 0 & 1 & 1 \\
0 & 0 & 0 & 1 & 0 & -1 & 0 \\
0 & 0 & 0 & 0 & 1 & 0 & 0 \\
0 & 0 & 0 & 0 & 0 & 1 & 0 \\
0 & 0 & 0 & 0 & 0 & 0 & 1
\end{array}\right] \text {, with inverse } W=\left[\begin{array}{rrrrrrr}
0 & -1 & -1 & -1 & 1 & 0 & 0 \\
-1 & 1 & 0 & 0 & 0 & 0 & 0 \\
2 & -2 & -1 & 0 & 0 & 1 & 1 \\
\hline 0 & 0 & 0 & 1 & 0 & 1 & 0 \\
0 & 0 & 0 & 0 & 1 & 0 & 0 \\
0 & 0 & 0 & 0 & 0 & 1 & 0 \\
0 & 0 & 0 & 0 & 0 & 0 & 1
\end{array}\right] .
$$

This unimodular multiplier has the required shape (25) to apply the parameter reduction scheme of Section 7.1. The reduced system is obtained by applying the substitution read from the inverse $W$ while the new variables are read from the unimodular multiplier $V$ itself.

The normal unimodular multiplier (Theorem 2.3) is

$$
V=\left[\begin{array}{rrr|rrrr}
-1 & 1 & 1 & -1 & 1 & -1 & -1 \\
-1 & 2 & 1 & -1 & 1 & -1 & -1 \\
0 & -2 & -1 & 0 & 0 & 1 & 1 \\
0 & 0 & 0 & 1 & 0 & 0 & 0 \\
0 & 0 & 0 & 0 & 1 & 0 & 0 \\
0 & 0 & 0 & 0 & 0 & 1 & 0 \\
0 & 0 & 0 & 0 & 0 & 0 & 1
\end{array}\right] \text {, with inverse } W=\left[\begin{array}{rrrrrrr}
0 & -1 & -1 & -1 & 1 & 0 & 0 \\
-1 & 1 & 0 & 0 & 0 & 0 & 0 \\
2 & -2 & -1 & 0 & 0 & 1 & 1 \\
\hline 0 & 0 & 0 & 1 & 0 & 0 & 0 \\
0 & 0 & 0 & 0 & 1 & 0 & 0 \\
0 & 0 & 0 & 0 & 0 & 1 & 0 \\
0 & 0 & 0 & 0 & 0 & 0 & 1
\end{array}\right] \text {. }
$$

\footnotetext{
${ }^{5}$ Here the Hermite normal form is $A \cdot V=\left[I_{3}, 0\right]$ by Proposition 5.1.
} 
This normal unimodular multiplier is obtained from the former simply by adding column 4 to column 6 . It leads to the reduced system

$$
\frac{d u}{d \tau}=u(1-v), \quad \frac{d v}{d \tau}=v(u-\alpha)
$$

with the substitution, which can be read from $W$,

$$
a \mapsto 1, k_{1} \mapsto 1, k_{2} \mapsto 1, k_{3} \mapsto \alpha, t \mapsto \tau, x \mapsto u, y \mapsto v
$$

and where the new variables, which are read on $V$,

$$
\alpha=\frac{k_{3}}{a k_{1}}, \quad \tau=a k_{1} t, \quad u=\frac{k_{2}}{a k_{1}} x, \quad v=\frac{k_{2}}{a k_{1}} y
$$

On this example we have thus illustrated how the choice of a unimodular multiplier affects the new variables and the reduced system. We do not claim that the normalization of the unimodular multiplier we offered is the best choice. Yet this is a choice that detects when the scaling symmetry can be fully used to reduce the number of parameters. The scheme proposed in Section 7.1, or even of Section 6, can nevertheless be put into action with other choices of unimodular multiplier.

Also, one should remark that the normalization of the unimodular multiplier, and hence the invariants we use as new variables, depends on the order of the parameters and variables. As a practical tip, one should choose an order where the parameters that we want to be substituted by 1 come first.

\subsection{Schackenberg model for a simple chemical reaction with limit cycle}

Consider the following dynamical system which models a plausible chemical reaction [20, Section 7.4] with parameters are $c=(a, b, k, h)$ :

$$
\begin{aligned}
& \frac{d x}{d t}=a-k x+h x^{2} y \\
& \frac{d y}{d t}=b-h x^{2} y .
\end{aligned}
$$

The scaling symmetries of this system are the scalings that leave $\left[a t x^{-1}-k t+h t x y, b t y^{-1}-h t x^{2}\right]$ invariant. Following Section 5 we form the matrix $K$ of the exponents appearing in those Laurent polynomials:

$$
K=\left[\begin{array}{ccccc}
0 & 1 & 0 & 0 & 0 \\
0 & 0 & 1 & 0 & 1 \\
1 & 0 & 0 & 0 & 0 \\
0 & 0 & 0 & 1 & 0 \\
1 & 1 & 1 & 1 & 1 \\
-1 & 0 & 1 & 0 & 2 \\
0 & 0 & 1 & -1 & 0
\end{array}\right]
$$

Applying Proposition 5.1 one then determines the matrix $A$ that describes the scaling symmetries for this system:

$$
A=\left[\begin{array}{rrrrrrr}
1 & 1 & 1 & 1 & -1 & 0 & 0 \\
0 & 0 & -1 & -3 & 1 & 1 & 1
\end{array}\right]
$$


The normal unimodular multiplier $V$ for the Hermite normal form of $A$ has the particular simple form (25). Together with its inverse $W$, they are given by:

$$
V=\left[\begin{array}{rr|rr|r|rr}
1 & 1 & -1 & 2 & 0 & -1 & -1 \\
0 & 0 & 1 & 0 & 0 & 0 & 0 \\
0 & -1 & 0 & -3 & 1 & 1 & 1 \\
0 & 0 & 0 & 1 & 0 & 0 & 0 \\
\hline 0 & 0 & 0 & 0 & 1 & 0 & 0 \\
\hline 0 & 0 & 0 & 0 & 0 & 1 & 0 \\
0 & 0 & 0 & 0 & 0 & 0 & 1
\end{array}\right], \quad W=\left[\begin{array}{rrrr|r|rr}
1 & 1 & -1 & 1 & -1 & 0 & 0 \\
0 & 0 & -1 & -3 & 1 & 1 & 1 \\
\hline 0 & 1 & 0 & 0 & 0 & 0 & 0 \\
0 & 0 & 0 & 1 & 0 & 0 & 0 \\
\hline 0 & 0 & 0 & 0 & 1 & 0 & 0 \\
\hline 0 & 0 & 0 & 0 & 0 & 1 & 0 \\
0 & 0 & 0 & 0 & 0 & 0 & 1
\end{array}\right] .
$$

The reduced model has hence two parameters $(\mathfrak{b}, \mathfrak{h})$ and two state variables $(\mathfrak{x}, \mathfrak{y})$ :

$$
\begin{aligned}
\frac{d \mathfrak{x}}{d \mathfrak{t}} & =1-\mathfrak{x}+\mathfrak{h} \mathfrak{x}^{2} \mathfrak{y} \\
\frac{d \mathfrak{y}}{d \mathfrak{t}} & =\mathfrak{b}-\mathfrak{h} \mathfrak{x}^{2} \mathfrak{y} .
\end{aligned}
$$

It is obtained by the substitution:

$$
a \mapsto 1, b \mapsto \mathfrak{b}, k \mapsto 1, h \mapsto \mathfrak{h}, t \mapsto \mathfrak{t}, x \mapsto \mathfrak{x}, y \mapsto \mathfrak{y} .
$$

If $(\mathfrak{b}, \mathfrak{h}, \mathfrak{x}(\mathfrak{t}), \mathfrak{y}(\mathfrak{t}))$ is a solution of this reduced system and $(\mathfrak{a}, \mathfrak{k})$ is any pair of constants then

$$
\left(\mathfrak{a}, \mathfrak{a} \mathfrak{k}, \frac{1}{\mathfrak{a} \mathfrak{b}}, \frac{\mathfrak{a} \mathfrak{h}}{\mathfrak{b}^{3}}, \mathfrak{b} \mathfrak{x}\left(\frac{\mathfrak{a}}{\mathfrak{b}} t\right), \mathfrak{b} \mathfrak{y}\left(\frac{\mathfrak{a}}{\mathfrak{b}} t\right)\right)
$$

is a solution of the original system. We have intrinsically considered the invariant variables:

$$
(\mathfrak{b}, \mathfrak{h})=\mathfrak{c}=\left(\frac{b}{a}, \frac{a^{2} h}{k^{3}}\right), \quad \mathfrak{t}=k t, \quad \text { and }(\mathfrak{x}, \mathfrak{y})=\mathfrak{z}=\left(\frac{k}{a}, \frac{k}{a}\right) \star(x, y) .
$$

Two remarks are in order for this example. First, had we chosen to order the variables as $c=(a, k, b, h)$, the normal unimodular multiplier would be of the form (11). Nonetheless, with the slightly more general form (25) we do obtain a model reduction as expected, without having to fiddle with the parameter order.

Secondly, the nondimensional model used in [20, Section 7.4] is

$$
\begin{aligned}
\frac{d \mathfrak{x}}{d t} & =\mathfrak{a}-\mathfrak{x}+\mathfrak{x}^{2} \mathfrak{y}, \\
\frac{d \mathfrak{y}}{d t} & =\mathfrak{b}-\mathfrak{x}^{2} \mathfrak{y} .
\end{aligned}
$$

It is obtained with the non dimensional variables

$$
\mathfrak{a}=\frac{h^{\frac{1}{2}}}{k^{\frac{3}{2}}} a, \mathfrak{b}=\frac{h^{\frac{1}{2}}}{k^{\frac{3}{2}}} b, \mathfrak{t}=k t, \mathfrak{x}=\frac{h^{\frac{1}{2}}}{k^{\frac{1}{2}}} x, \mathfrak{y}=\frac{h^{\frac{1}{2}}}{k^{\frac{1}{2}}} y .
$$

To cast this in the context of symmetry reduction, we can resort to the general approach of $[6,9,18]$. The variety of $(h-1, k-1)$ is a quasi-section: it has two points of intersection with a generic orbit. The related replacement invariants are thus algebraic functions of degree 2 and hence the appearance of square roots. Here, the state variables $x$ and $y$ are molecular concentrations of reactants that evolve with time, while $a$ and $b$ reflect constant supply of some of the reactants. The parameters $k$ and $h$ are stochiometric coefficients. As such, they are positive and the use of square roots is well defined. The reductions obtained with the approach proposed in this paper are always rational. We therefore do not need to argue about the sign of the parameters or of the state variables. 


\subsection{Prey-predator model}

Consider the predator-prey model given by the dynamical system

$$
\begin{aligned}
& \frac{d n}{d t}=n\left(r\left(1-\frac{n}{K}\right)-k \frac{p}{n+d}\right), \\
& \frac{d p}{d t}=s p\left(1-\frac{h p}{n}\right)
\end{aligned}
$$

which appears in the introduction. The parameters are $c=(r, h, K, s, k, d)$ and the state variables are $z=(n, p)$.

The scaling symmetry of this system was determined in Example 5.2 and is given by

$$
A=\left[\begin{array}{rrrrrrrrr}
-1 & 0 & 0 & -1 & -1 & 0 & 1 & 0 & 0 \\
0 & 1 & 1 & 0 & 1 & 1 & 0 & 1 & 0 \\
0 & -1 & 0 & 0 & -1 & 0 & 0 & 0 & 1
\end{array}\right]
$$

The normal unimodular multiplier $V$ for the Hermite normal form of $A$ has the particular simple form (25). Together with its inverse $W$, they are given by:

$$
V=\left[\begin{array}{rrr|rrr|r|rr}
-1 & 0 & 0 & -1 & -1 & 0 & 1 & 0 & 0 \\
0 & 0 & -1 & 0 & -1 & 0 & 0 & 0 & 1 \\
0 & 1 & 1 & 0 & 0 & -1 & 0 & -1 & -1 \\
0 & 0 & 0 & 1 & 0 & 0 & 0 & 0 & 0 \\
0 & 0 & 0 & 0 & 1 & 0 & 0 & 0 & 0 \\
0 & 0 & 0 & 0 & 0 & 1 & 0 & 0 & 0 \\
\hline 0 & 0 & 0 & 0 & 0 & 0 & 1 & 0 & 0 \\
\hline 0 & 0 & 0 & 0 & 0 & 0 & 0 & 1 & 0 \\
0 & 0 & 0 & 0 & 0 & 0 & 0 & 0 & 1
\end{array}\right], \quad W=\left[\begin{array}{rrrrrr|r|rr}
-1 & 0 & 0 & -1 & -1 & 0 & 1 & 0 & 0 \\
0 & 1 & 1 & 0 & 1 & 1 & 0 & 1 & 0 \\
0 & -1 & 0 & 0 & -1 & 0 & 0 & 0 & 1 \\
\hline 0 & 0 & 0 & 1 & 0 & 0 & 0 & 0 & 0 \\
0 & 0 & 0 & 0 & 1 & 0 & 0 & 0 & 0 \\
0 & 0 & 0 & 0 & 0 & 1 & 0 & 0 & 0 \\
\hline 0 & 0 & 0 & 0 & 0 & 0 & 1 & 0 & 0 \\
\hline 0 & 0 & 0 & 0 & 0 & 0 & 0 & 1 & 0 \\
0 & 0 & 0 & 0 & 0 & 0 & 0 & 0 & 1
\end{array}\right] .
$$

The reduced system

$$
\begin{aligned}
\frac{d \mathfrak{n}}{d \mathfrak{t}} & =\mathfrak{n}\left((1-\mathfrak{n})-\mathfrak{k} \frac{\mathfrak{p}}{\mathfrak{n}+\mathfrak{d}}\right), \\
\frac{d \mathfrak{p}}{d \mathfrak{t}} & =\mathfrak{s} \mathfrak{p}\left(1-\frac{\mathfrak{p}}{\mathfrak{n}}\right) .
\end{aligned}
$$

is then obtained by the substitution: $r \mapsto 1, h \mapsto 1, K \mapsto 1, s \mapsto \mathfrak{s}, k \mapsto \mathfrak{k}, d \mapsto \mathfrak{d}, t \mapsto \mathfrak{t}, n \mapsto \mathfrak{n}, p \mapsto \mathfrak{p}$.

If $(\mathfrak{s}, \mathfrak{k}, \mathfrak{d}, \mathfrak{n}(\mathfrak{t}), \mathfrak{p}(\mathfrak{t}))$ is a solution of this reduced system and $(\mathfrak{a}, \mathfrak{b}, \mathfrak{c})$ is any triplet of constants then

$$
\left(\frac{1}{\mathfrak{a}}, \frac{\mathfrak{b}}{\mathfrak{c}}, \mathfrak{b}, \frac{\mathfrak{s}}{\mathfrak{a}}, \frac{\mathfrak{b}}{\mathfrak{a} \mathfrak{c}} \mathfrak{k}, \mathfrak{b} \mathfrak{d}, \mathfrak{b} \mathfrak{n}\left(\frac{t}{\mathfrak{a}}\right), \mathfrak{c} \mathfrak{p}\left(\frac{t}{\mathfrak{a}}\right)\right)
$$

is a solution of the original system. The reduced system is actually the dynamics for the invariant variables:

$$
(\mathfrak{s}, \mathfrak{k}, \mathfrak{d})=\mathfrak{c}=\left(\frac{s}{r}, \frac{k}{r h}, \frac{d}{K}\right), \quad \mathfrak{t}=r t, \quad \text { and }(\mathfrak{n}, \mathfrak{p})=\mathfrak{z}=\left(\frac{1}{K}, \frac{h}{K}\right) \star(n, p) .
$$

\section{Conclusion and prospects}

In this paper we have made use of the Hermite normal form of the matrix of exponents of a scaling. We showed how a scaling symmetry can be determined and used algorithmically to reduce dynamical systems 
rationally. We demonstrated how this in particular applies to the reduction of the number of parameters in models of mathematical biology. All the algorithms in this paper have been implemented in the computer algebra system Maple with the code available from the authors.

There are a number of research topics that we plan to consider for future work. We have used the Hermite normal form and a normalized unimodular multiplier as the basic algorithmic tools for computing scaling symmetries, rational invariants and reductions. However other normal forms and normalizations for the unimodular multiplier can also be appropriate for such computations. It nonetheless remains an open question as to which rank revealing normal form and unimodular multiplier normalization are in fact best for a given application.

We are also interested in scaling symmetry reductions for more general differential systems. In this paper we treated the simplest case of evolution equation, one with a single independent variable. However evolution equations in terms of partial differential equations are also common in physics and mathematical biology. We would like to obtain an algorithmic parameter reduction technique as explicit as that presented in Section 7 for such situations. A general scheme of scaling symmetry reduction of higher order (partial) differential systems as explicit as found for dynamical system in Section 6 is a challenge.

\section{References}

[1] I. M. Anderson and M. E. Fels. Exterior differential systems with symmetry. Acta Appl. Math., 87(1$3): 3-31,2005$.

[2] B. Beckermann, G. Labahn, and G. Villard. Normal forms for general polynomial matrices. Journal of Symbolic Computation, 41(6):708-737, 2006.

[3] G. Birkhoff. Hydrodynamics: A study in logic, fact and similitude. Princeton Univ. Press, 1960.

[4] P. Bridgman. Dimensional Analysis. Yale Univ. Press, 1931.

[5] H. Cohen. A Course in Computational Algebraic Number Theory. Springer-Verlag, 1993.

[6] M. Fels and P. Olver. Moving coframes. ii. Regularization and theoretical foundations. Acta Appl. Math., 55(2):127-208, 1999.

[7] M. E. Fels. Integrating scalar ordinary differential equations with symmetry revisited. Found. Comput. Math., 7(4):417-454, 2007.

[8] G. Havas, B. Majewski, and K. Matthews. Extended gcd and Hermite normal form algorithms via lattice basis reduction. Experimental Mathematics, 7(2), 1998.

[9] E. Hubert and I. Kogan. Rational invariants of a group action. Construction and rewriting. Journal of Symbolic Computation, 42(1-2):203-217, 2007.

[10] E. Hubert and I. Kogan. Smooth and algebraic invariants of a group action. Local and global constructions. Foundations of Computational Mathematics, 7(4), 2007.

[11] E. Hubert and A. Sedoglavic. Polynomial Time Nondimensionalisation of Ordinary Differential Equations via their Lie Point Symmetries, 2006.

[12] H. E. Huntley. Dimensional Analysis. Dover Publications, New York, 1967.

[13] Y. Ishida. Formula processing on physical systems. Complex Systems, 11(2):141-160, 1997.

[14] G. Kemper. The computation of invariant fields and a new proof of a theorem by Rosenlicht. Transformation Groups, 12:657-670, 2007. 
[15] R. Khanin. Dimensional analysis in computer algebra. In Proceedings of ISSAC 2001. ACM press, 2001.

[16] F. Lemaire and A. Ürgüplü. A method for semi-rectifying algebraic and differential systems using scaling type Lie point symmetries with linear algebra. In Proceedings of ISSAC 2010. ACM press, 2010.

[17] C. Lin and L. Segel. Mathematics applied to deterministic problems in the natural sciences. Society for Industrial and Applied Mathematics, 1988.

[18] E. Mansfield. A Practical Guide to the Invariant Calculus. Cambridge University Press, 2010.

[19] J. Müller-Quade and T. Beth. Calculating generators for invariant fields of linear algebraic groups. In Applied algebra, algebraic algorithms and error-correcting codes, volume 1719 of LNCS. Springer, 1999.

[20] J. D. Murray. Mathematical Biology, volume 17 of Interdisciplinary Applied Mathematics. Springer, 2002.

[21] P. J. Olver. Applications of Lie Groups to Differential Equations. Number 107 in Graduate texts in Mathematics. Springer-Verlag, New York, 1986.

[22] V. L. Popov and E. B. Vinberg. Invariant Theory. In Algebraic geometry. IV, Encyclopaedia of Mathematical Sciences. Springer-Verlag, 1994.

[23] A. Schrijver. Theory of Linear and Integer Programming. Wiley, 1986.

[24] A. Sedoglavic. Reduction of algebraic parametric systems by rectification of their affine expanded lie symmetries. In Algebraic Biology, volume 4545 of LNCS. Springer, 2007.

[25] B. Sturmfels. Gröbner Bases and Convex Polytopes. American Mathematical Society, Providence, RI, 1996. 